OPEN ACCESS

Edited by:

Yuji Morita,

Aichi Gakuin University, Japan

Reviewed by:

Lars Jelsbak,

Technical University of Denmark,

Denmark

Sabine Delannoy,

Agence Nationale de Sécurité

Sanitaire de l'Alimentation, de

l'Environnement et du Travail

(ANSES), France

Joanna Goldberg,

Emory University School of Medicine,

United States

*Correspondence:

Isabel Sá-Correia

isacorreia@tecnico.ulisboa.pt

${ }^{\dagger}$ These authors have contributed equally to this work

Specialty section:

This article was submitted to

Infectious Diseases,

a section of the journal

Frontiers in Microbiology

Received: 20 March 2017

Accepted: 22 May 2017

Published: 08 June 2017

Citation:

Hassan AA, Maldonado RF dos Santos SC, Di Lorenzo F, Silipo A, Coutinho CP, Cooper VS,

Molinaro A, Valvano MA and

Sá-Correia I (2017) Structure of O-Antigen and Hybrid Biosynthetic Locus in Burkholderia cenocepacia Clonal Variants Recovered from a Cystic Fibrosis Patient.

Front. Microbiol. 8:1027. doi: 10.3389/fmicb.2017.01027

\section{Structure of O-Antigen and Hybrid Biosynthetic Locus in Burkholderia cenocepacia Clonal Variants Recovered from a Cystic Fibrosis Patient}

\author{
A. A. Hassan ${ }^{1 t}$, Rita F. Maldonado ${ }^{11}$, Sandra C. dos Santos ${ }^{1 \dagger}$, Flaviana Di Lorenzo ${ }^{21}$, \\ Alba Silipo², Carla P. Coutinho', Vaughn S. Cooper ${ }^{3}$, Antonio Molinaro', \\ Miguel A. Valvano ${ }^{4}$ and Isabel Sá-Correia ${ }^{1 *}$
}

\begin{abstract}
' Department of Bioengineering, Institute for Bioengineering and Biosciences, Instituto Superior Técnico, Universidade de Lisboa, Lisboa, Portugal, ${ }^{2}$ Department of Chemical Sciences, University of Napoli Federico Il Complesso Universitário Monte Santangelo, Napoli, Italy, ${ }^{3}$ Department of Microbiology and Molecular Genetics, University of Pittsburgh School of Medicine, Pittsburgh, PA, United States, ${ }^{4}$ The Wellcome-Wolfson Institute for Experimental Medicine, Queen's University Belfast, Belfast, United Kingdom
\end{abstract}

Burkholderia cenocepacia is an opportunistic pathogen associated with chronic lung infections and increased risk of death in patients with cystic fibrosis (CF). In this work, we investigated the lipopolysaccharide (LPS) of clinical variants of $B$. cenocepacia that were collected from a CF patient over a period of 3.5 years, from the onset of infection until death by necrotizing pneumonia (cepacia syndrome). We report the chemical structure of the LPS molecule of various sequential isolates and the identification of a novel hybrid O-antigen $(\mathrm{OAg})$ biosynthetic cluster. The OAg repeating unit of the LPS from IST439, the initial isolate, is a $[\rightarrow 2)-\beta$-D-Ribf- $(1 \rightarrow 4)-\alpha-D-G a l p N A c-(1 \rightarrow]$ disaccharide, which was not previously described in B. cenocepacia. The IST439 OAg biosynthetic gene cluster contains 7 of 23 genes that are closely homologous to genes found in B. multivorans, another member of the Burkholderia cepacia complex. None of the subsequent isolates expressed OAg. Genomic sequencing of these isolates enabled the identification of mutations within the OAg cluster, but none of these mutations could be associated with the loss of OAg. This study provides support to the notion that OAg LPS modifications are an important factor in the adaptation of $B$. cenocepacia to chronic infection and that the heterogeneity of OAgs relates to variation within the OAg gene cluster, indicating that the gene cluster might have been assembled through multiple horizontal transmission events.

\footnotetext{
Keywords: Burkholderia cepacia complex, cystic fibrosis, chronic infection, O-antigen, lipopolysaccharide, clonal variation
}

\section{INTRODUCTION}

Burkholderia cenocepacia is a Gram-negative opportunistic human pathogen of the Burkholderia cepacia complex (Bcc), relevant in immunocompromised individuals and cystic fibrosis (CF) patients (Mahenthiralingam et al., 2002, 2005). B. cenocepacia lung infections in CF patients are associated with poor prognosis and increased risk of death (Mahenthiralingam et al., 2002, 2005; 
Drevinek and Mahenthiralingam, 2010). In comparison to Pseudomonas aeruginosa, the major CF pathogen, less is known about the molecular mechanisms involved in the adaptation of B. cenocepacia to the CF lung (Coutinho et al., 2011b; Maldonado et al., 2016).

The lipopolysaccharide (LPS) O-antigen (OAg) biosynthetic cluster is under strong selective pressure during chronic infection (reviewed in Maldonado et al., 2016). LPS is a major component of the Gram-negative bacterial outer membrane, which participates in host-bacterium interactions, such as adhesion, immune evasion, persistence, and antimicrobial resistance (Raetz and Whitfield, 2002; De Soyza et al., 2008; Valvano et al., 2011; Maldonado et al., 2016). LPS consists of a core oligosaccharide (core) that is covalently linked to a lipophilic glycan termed lipid A (Whitfield and Trent, 2014). In many bacteria, the LPS is extended by an OAg polysaccharide that is linked to the core. The lipid $A$ is made of a $\beta$ - $(1 \rightarrow 6)$ glucosamine disaccharide acylated with primary fatty acids at positions 2 and 3 of both glucosamine residues, which are in turn phosphorylated at the 1 - and $4^{\prime}$-positions. Secondary acyl chains can further substitute primary fatty acids at their hydroxyl positions (Whitfield and Trent, 2014). The core, subdivided into inner and outer core, comprises conserved monosaccharide residues, such as heptoses and 3-deoxy-Dmanno-oct-2-ulosonic acid (Kdo), which are typically unique to the LPS molecule (Whitfield and Trent, 2014). In Burkholderia species, one of the Kdo residues is modified to D-glycero-Dtalo-oct-2-ulosonic acid (Ko) (Silipo et al., 2005). The OAg extends away from the outer membrane surface becoming exposed to the extracellular milieu; it is composed of linear or branched homo- or heteropolysaccharides of variable length, with subunits consisting of up to eight different sugars (Valvano et al., 2011).

Recent studies at genome (Spencer et al., 2003; Lieberman et al., 2011; Traverse et al., 2013), transcriptome (Mira et al., 2011), and proteome (Madeira et al., 2011, 2013) levels indicate that the LPS and particularly the OAg undergo alterations during chronic infection, which could be attributed to bacterial adaptation to biofilm lifestyle (Lieberman et al., 2011; Traverse et al., 2013), immune evasion and selective pressure from antibiotics (reviewed in Maldonado et al., 2016). The airways of CF patients are colonized by polymicrobial communities that show variability in composition and diversity (Coburn et al., 2015). The CF microbiota is also exposed to a fluctuating environment and multiple selective pressures arising from nearly constant antibiotic treatment, oxygen limitation, and the persistent host inflammatory response (Döring et al., 2011; Cullen and McClean, 2015). Therefore, infecting bacteria give rise to genetically heterogeneous populations in which different phenotypes adaptive for growth and survival are selected (Harrison, 2007; Yang et al., 2011). Understanding the adaptive and evolutionary mechanisms within chronic infections of the CF airway may help improve the management of these infections.

We investigated a collection of serial clonal isolates of B. cenocepacia obtained from a CF patient over a period of 3.5-years since the onset of infection until the patient's death with the cepacia syndrome (Cunha et al., 2003; Coutinho et al., 2011a). These isolates belong to the epidemic ET-12 lineage (represented by the prototypic strains J2315 and K56-2) (Mahenthiralingam et al., 2000; Holden et al., 2009). This patient was also co-infected with $B$. multivorans, another member of the Bcc, which appeared before the isolation of the early B. cenocepacia strain (isolate IST439); co-infection continued until near the patient's death (Cunha et al., 2003). The B. cenocepacia isolates from this patient were characterized by phenotypic (Coutinho et al., 2011a), transcriptomic (Mira et al., 2011), proteomic (Madeira et al., 2011, 2013) and metabolic profiling (Moreira et al., 2016a), as well as by comparative genomics (still unpublished). Isolates of particular interest are B. cenocepacia IST4113 (a highly antibiotic resistant variant retrieved after an exacerbation treated with intravenous therapy), IST4134 (obtained just before the patient's death), and IST4129 (a variant that exhibits attenuated virulence related with the loss of the third replicon (Moreira et al., 2016b). The virulence potential of these isolates in non-mammalian host models (Moreira et al., 2016b) and their ability to modulate dendritic cell function were also compared (Cabral et al., 2017).

In this study, we analyzed the chemical structure of the LPS molecule and the genetic organization of the predicted OAg biosynthetic cluster in these serial isolates. Our results reveal that the early $B$. cenocepacia IST439 isolate encodes a functional genetic cluster responsible for OAg biosynthesis, with a hybrid composition including genes highly homologous to $B$. multivorans genes. Further, this isolate produces a structurally different OAg from that previously reported in the ET12 lineage strains, while all subsequent $B$. cenocepacia isolates lost the ability to produce an OAg molecule.

\section{MATERIALS AND METHODS}

\section{Bacterial Strains and Growth Conditions}

The isolates investigated in this study are indicated in Table $\mathbf{1}$ (Cunha et al., 2003; Coutinho et al., 2011a). B. cenocepacia and $B$. multivorans isolates were recovered from the sputum of a CF patient at the major Portuguese CF Center in Hospital de Santa Maria (HSM), from Centro Hospitalar Lisboa Norte EPE, Lisbon, Portugal, between 1998 and 2002. These isolates belong to the same clonal complex and ET-12 lineage (Moreira et al., 2016b). Studies involving these isolates were approved by the ethics committee of the Hospital, and the anonymity of the patient was preserved. The genome of the soil strain B. multivorans ATCC 17616 (NCBI nucleotide accession number: NC_010804.1) (Vandamme et al., 1997) was used for comparisons. Bacterial cultures were stored at $-80^{\circ} \mathrm{C}$ in 1:1 (v/v) glycerol. Bacteria were grown in LB Lennox (LB; Conda, Pronadisa) at $37^{\circ} \mathrm{C}$ with shaking at $250 \mathrm{rpm}$ or in LB agar plates. Escherichia coli strains (Table 1) were grown in the same conditions. When needed, growth media were supplemented with antibiotics at the following concentrations: for B. cenocepacia, trimethoprim (TMP) at $100 \mu \mathrm{g} / \mathrm{ml}$, and for E. coli, TMP at $50 \mu \mathrm{g} / \mathrm{ml}$ and kanamycin (KAN) at $40 \mu \mathrm{g} / \mathrm{ml}$. 
TABLE 1 | Description of Burkholderia isolates and E. coli strains used in this study.

\begin{tabular}{|c|c|c|c|}
\hline Bacterial isolate & Isolation date & Species & Description \\
\hline IST419 & 2/26/1998 & B. multivorans & Clinical isolates obtained from a chronically infected patient followed at the Cystic Fibrosis \\
\hline IST439 & $1 / 30 / 1999$ & B. cenocepacia IIIA_ET-12 & Center of Hospital Santa Maria, Lisbon, Portugal (Cunha et al., 2003; \\
\hline IST4103 & $7 / 24 / 2001$ & B. cenocepacia IIIA_ET-12 & Coutinho et al., 2011a; Moreira et al., 2016b) \\
\hline IST4110 & $9 / 25 / 2001$ & B. cenocepacia IIIA_ET-12 & \\
\hline IST4112 & $10 / 11 / 2001$ & B. cenocepacia IIIA_ET-12 & \\
\hline IST4113 & $11 / 6 / 2001$ & B. cenocepacia IIIA_ET-12 & \\
\hline IST4116A & 2/11/2002 & B. cenocepacia IIIA_ET-12 & \\
\hline IST4116B & 2/11/2002 & B. cenocepacia IIIA_ET-12 & \\
\hline IST4131 & 2/26/2002 & B. cenocepacia IIIA_ET-12 & \\
\hline IST4129 & $3 / 26 / 2002$ & B. cenocepacia IIIA_ET-12 & \\
\hline IST4130 & $5 / 14 / 2002$ & B. cenocepacia IIIA_ET-12 & \\
\hline IST4134 & $7 / 2 / 2002$ & B. cenocepacia IIIA_ET-12 & \\
\hline ATCC 17616 & & B. multivorans & Soil isolate (Berkeley, CA, United States) (Vandamme et al., 1997; Nishiyama et al., 2010) \\
\hline DH5- $\alpha$ (pRK2013) & & E. coli & Helper strain for triparental conjugation (Figurski and Helinski, 1979) \\
\hline ER2925 (dam- dcm-) & & E. coli & Host for plasmids used to transform resilient Burkholderia strains (Craig et al., 1989) \\
\hline
\end{tabular}

\section{LPS Extraction, Purification, and Compositional Analyses}

Single-colony purified cells of the early isolate (IST439) and three late-stage clonal variants (IST4113, IST4129, and IST4134) were used for LPS purification and structural analysis. Bacteria were first grown overnight in LB broth until mid-exponential phase at $37^{\circ} \mathrm{C}$ with shaking $(250 \mathrm{rpm})$. Cultures were diluted to an $\mathrm{OD}_{640} \mathrm{~nm}$ of 0.2 , and $100 \mu \mathrm{l}$ of the cellular suspensions were plated onto LB agar plates and incubated for $24 \mathrm{~h}$ at $37^{\circ} \mathrm{C}$. Bacteria were scraped from the agar surface, collected, autoclaved, and lyophilized. LPS from bacterial dried cells was extracted by the hot phenol/water method (Westphal and Jann, 1965). The nature of the extracted material was checked by SDS-PAGE after gel silver staining (Kittelberger and Hilbink, 1993). To remove contaminants the extracts were treated with RNase (Roth, Germany), DNase (Roth, Germany) and Proteinase K (Roth, Germany) at 37 and $56^{\circ} \mathrm{C}$, followed by dialysis against distilled water. The LPS was further purified by ultracentrifugation $\left(4^{\circ} \mathrm{C}, 30,000 \mathrm{rpm}, 24 \mathrm{~h}\right)$ and gel-filtration chromatography. The monosaccharide content of the sample was determined by analysis of the acetylated O-methyl glycoside derivatives after treatment with $\mathrm{HCl} / \mathrm{MeOH}\left(1.25 \mathrm{M}, 85^{\circ} \mathrm{C}\right.$, $24 \mathrm{~h})$ plus acetylation with acetic anhydride in pyridine $\left(85^{\circ} \mathrm{C}\right.$, $30 \mathrm{~min}$ ) using gas-liquid chromatography mass spectrometry (GLC-MS). The sugar linkages were determined as described (Ciucanu and Kerek, 1984). The total fatty acid content was determined on intact LPS by treatment with $4 \mathrm{M} \mathrm{HCl}\left(100^{\circ} \mathrm{C}\right.$, $4 \mathrm{~h})$, followed by $5 \mathrm{M} \mathrm{NaOH}\left(100^{\circ} \mathrm{C}, 30 \mathrm{~min}\right)$. After extraction in chloroform, fatty acids were methylated with diazomethane and analyzed by GLC-MS. The ester bound fatty acids were released by base-catalyzed hydrolysis with aqueous $\mathrm{NaOH} 0.5 \mathrm{M}, \mathrm{MeOH}$ $\left(1: 1, \mathrm{v} / \mathrm{v}, 85^{\circ} \mathrm{C}, 2 \mathrm{~h}\right)$, and then the product was acidified, extracted in chloroform, methylated with diazomethane, and analyzed by GLC-MS. The absolute configuration of fatty acids was determined as previously described (Rietschel, 1976). Authentic 3-hydroxy fatty acids were used to assign the (R) configuration to all LPS/LOS acyl chains.

\section{NMR Spectroscopy}

Prior to NMR spectroscopy an aliquot of the purified LPS $(20 \mathrm{mg})$ was hydrolyzed with acetate buffer $\left(100^{\circ} \mathrm{C}, 2 \mathrm{~h}\right)$. After centrifugation, the supernatant, containing the saccharide fraction, was collected, lyophilized, and purified by size exclusion chromatography. 1D and $2 \mathrm{D}{ }^{1} \mathrm{H}$ NMR spectra were recorded on a Bruker 600 DRX equipped with a cryo probe. The solvent employed was $\mathrm{D}_{2} \mathrm{O}$ and the temperature was $298 \mathrm{~K}$ and $\mathrm{pD}$ was 7 . Spectra calibration was performed with internal acetone $\left(\delta_{\mathrm{H}} 2.225 \mathrm{ppm}, \delta_{\mathrm{C}} 31.45 \mathrm{ppm}\right)$. The double-quantum filtered phase sensitive correlation spectroscopy (DQF-COSY) experiment was executed by using data sets of $4096 \times 256$ points. Total correlation spectroscopy (TOCSY) experiments were carried out with spinlock times of $100 \mathrm{~ms}$, using data sets $(\mathrm{t} 1 \times \mathrm{t} 2)$ of $4096 \times 256$ points. Rotating frame Overhauser enhancement spectroscopy (ROESY) and Nuclear Overhauser enhancement spectroscopy (NOESY) experiments were performed by using data sets $(\mathrm{t} 1 \times \mathrm{t} 2)$ of $4096 \times 256$ points and by using mixing times between 100 and $400 \mathrm{~ms}$. In all homonuclear experiments the data matrix was zero-filled in both dimensions to give a matrix of $4 \mathrm{~K} \times 2 \mathrm{~K}$ points and was resolution enhanced in both dimensions by a cosine-bell function before Fourier Transformation. The determination of coupling constants was obtained by 2D phase sensitive DQF-COSY (Piantini et al., 1982; Rance et al., 1983). Heteronuclear single quantum coherence $\left({ }^{1} \mathrm{H},{ }^{13} \mathrm{C}\right.$ HSQC $)$ and heteronuclear multiple bound correlation $\left({ }^{1} \mathrm{H},{ }^{13} \mathrm{C} \mathrm{HMBC}\right)$ experiments were recorded in ${ }^{1} \mathrm{H}$-detection mode by single-quantum coherence with protein decoupling in the ${ }^{13} \mathrm{C}$ domain using data sets of $2048 \times 256$ points. ${ }^{1} \mathrm{H},{ }^{13} \mathrm{C}$ HSQC was performed using sensitivity improvement and in the phase-sensitive mode using Echo/Antiecho gradient selection, with multiplicity editing during selection step (States et al., 1982). The ${ }^{1} \mathrm{H},{ }^{13} \mathrm{C}$ HMBC experiment was optimized on long-range coupling constants with low-pass $J$ filter to suppress one-bound connectivity, using gradient pulses for selection. A delay of $60 \mathrm{~ms}$ was employed for the evolution of long-range correlations. It was used a long-range coupling constant value of $6 \mathrm{~Hz}$. 
The data matrix in both heteronuclear experiments was extended to $2048 \times 1024$ points using forward linear prediction (Stern et al., 2002).

\section{MALDI Mass Spectrometry}

MALDI-TOF mass spectra of the intact LPS were recorded in reflectron mode and negative ion polarity on a Perseptive (Framingham, MA, United States) Voyager STR equipped with delayed extraction technology. Ions formed by a pulsed UV laser beam (nitrogen laser, $\lambda=337 \mathrm{~nm}$ ) were accelerated by $24 \mathrm{kV}$. LPS/LOS preparation was executed as described before (Sturiale et al., 2005; Lombardi et al., 2013).

\section{Small-Scale LPS Extraction for SDS-Polyacrylamide Gel Electrophoresis Analysis}

Lipopolysaccharide was extracted as previously described (Marolda et al., 1990) with small modifications. Briefly, Burkholderia isolates were harvested from overnight liquid cultures by centrifugation for $1 \mathrm{~min}$ after $\mathrm{OD}_{640 \mathrm{~nm}}$ adjustment to 2.0 in $1 \mathrm{ml}$ of PBS, suspended in $150 \mu \mathrm{l}$ of lysis buffer containing $2 \%$ SDS, 4\% 2- $\beta$-mercaptoethanol, and $500 \mathrm{mM}$ Tris- $\mathrm{HCl}$ ( $\mathrm{pH}$ 6.8), and boiled for $10 \mathrm{~min}$. Proteinase $\mathrm{K}(20 \mathrm{mg} / \mathrm{ml})$ was added, and the sample was incubated at $60^{\circ} \mathrm{C}$ for $2 \mathrm{~h}$. Finally, samples were mixed with the tracking dye solution (125 mM Tris-HCl [pH 6.8], 2\% SDS, 20\% [v/v] glycerol, $0.002 \%$ bromophenol blue, and $10 \%$ mercaptoethanol) and boiled for 5 min before the gels were loaded. LPS samples were resolved by electrophoresis (at $150 \mathrm{~V}$ for about $1 \mathrm{~h} 40 \mathrm{~m}$ ) in $14 \%$ polyacrylamide gels with a Tricine-SDS system followed by silver staining as previous described to visualize the banding patterns of the OAg (Marolda et al., 2006).

\section{Genomic DNA Sequencing, Assembly, and Annotation}

Bacterial cultures were prepared by suspending isolated colonies from LB agar plates in $3 \mathrm{~mL} \mathrm{LB}$ broth, followed by overnight growth at $37^{\circ} \mathrm{C}$ with shaking at $250 \mathrm{rpm}$. Genomic DNA was extracted and purified using a DNeasy Blood and Tissue Lit (Qiagen, Germany) according to manufacturer instructions. DNA concentration and purity were assessed using a Nanodrop ND-1000 spectrophotometer.

Burkholderia multivorans IST419 was sequenced on an Illumina NextSeq500 at the University of Pittsburgh, Pittsburgh, PA, United States. Raw fastq paired-end files were processed for removal of Illumina adapters, trimming, and qualitybased filtering using Trimmomatic (Bolger et al., 2014). De novo assembly was performed using Velvet with automated optimization of assembly parameters (Zerbino and Birney, 2008), using the two sets of pair-ended reads, and annotated using the prokaryotic genome annotation tool Prokka (Seemann, 2014). For the identification of mutational events in the OAg genetic clusters, read data sets were mapped against the relevant reference genomes (B. cenocepacia or B. multivorans) using BWA-MEM of BWA (Li and Durbin, 2010) and Bowtie 2
(Langmead and Salzberg, 2012), and visualized in IGV (Robinson et al., 2011).

DNA sequence reads for all isolates are available in the EMBL's European Nucleotide Archive (ENA) under accession number PRJEB20052 ${ }^{1}$ ). The sequence of the OAg cluster of B. cenocepacia IST439 is given as Supplementary Table S1.

\section{In Silico Characterization of O-Antigen Biosynthetic Gene Clusters}

Genome sequences of 11 B. cenocepacia sequential isolates and the sequence of $B$. multivorans IST419 obtained from a CF patient (Coutinho et al., 2011a) were used to investigate the OAg biosynthetic cluster (the detailed analysis of the remaining genomic differences will be reported elsewhere). The gene composition of the OAg biosynthetic gene cluster was determined by examining the region flanked by genes $a p a H$ and ureG (Ortega et al., 2005). These flanking genes were detected by BLASTN (Altschul et al., 1990) using as queries the gene sequences from B. cenocepacia J2315 against each of the corresponding nucleotide databases created from the available sequences under study. The assembled scaffolds of all studied isolates were cropped to obtain only the OAg cluster (apaH-ureG) for further examination. Computer-assisted analysis for these clusters was performed in Artemis (Carver et al., 2008) and by BLASTP (Altschul et al., 1990) to reveal the open reading frames (ORFs). Then, the clusters were visualized and compared with the OAg cluster of B. cenocepacia K56-2 (NCBI nucleotide accession number: NZ_ALJA00000000.2) by Artemis and ACT (Carver et al., 2008), and gene function was assigned based on the analysis of predicted ORF homologies.

\section{Molecular Cloning of wbil and bmul_2510 Genes in B. cenocepacia Clonal Variants Lacking O-Antigen}

Genes wbiI and bmul_2510 were PCR amplified from B. cenocepacia IST439 chromosomal DNA using a Hot Start High Fidelity polymerase (Qiagen) and cloned into pSCrhaB2 (Cardona and Valvano, 2005; Cardona et al., 2006). Primers were designed based on the genome sequence of IST439 and included specific restriction enzyme sites and a sequence encoding the FLAG epitope tag (Supplementary Table S2). Primers WbiI-flag-NdeI and WbiI_439_XbaI were used to clone wbiI fused to an N-terminal FLAG tag; primers wbiI_439_NdeI and Wbil-flag-XbaI were used to clone wbiI fused to a C-terminal FLAG tag; primers Bmul_2510-439-NdeI and Bmul_2510flag-XbaI were used to clone bmul_2510 fused to a C-terminal FLAG tag; primers P1, P2, P3 and P4 were used to clone both wbiI and bmul_2510 with C-terminal FLAGs using the Gibson Assembly strategy (New England BioLabs). The amplification conditions were $5 \mathrm{~min}$ at $95^{\circ} \mathrm{C}, 30$ cycles of $95^{\circ} \mathrm{C}$ for $30 \mathrm{~s}, 60^{\circ} \mathrm{C}$ for $1 \mathrm{~min}$, and $72^{\circ} \mathrm{C}$ for $2 \mathrm{~min}$, and a final extension of $10 \mathrm{~min}$ at $72^{\circ} \mathrm{C}$. The resulting products were digested with $\mathrm{NdeI}$ and XbaI, ligated to pSCrhaB2 and introduced into E. coli ER2925 (New England BioLabs) (Craig et al., 1989) by transformation.

\footnotetext{
${ }^{1}$ http://www.ebi.ac.uk/ena/data/view/PRJEB20052
} 
Transformants carrying recombinant plasmids with the DNA insert were screened by colony PCR with primers 824 and pSC rev, which anneal to vector sequences flanking the cloning sites. All constructs were confirmed by DNA sequencing.

\section{Plasmid Conjugation into B. cenocepacia}

Plasmids were mobilized by triparental mating (Craig et al., 1989) into all B. cenocepacia clonal variants lacking the OAg that possess single nucleotide polymorphisms (SNP) in Bmul_2510 and/or wbiI using E. coli DH5- $\alpha$ carrying the pRK2013 helper plasmid (Figurski and Helinski, 1979). Exconjugants were selected on LB agar plates supplemented with $100 \mu \mathrm{g} / \mathrm{ml}$ of TMP and $200 \mu \mathrm{g} / \mathrm{ml}$ of ampicillin (AMP).

\section{Western Blot}

Protein expression in $B$. cenocepacia clonal variants expressing wbiI or bmul_2510 from IST439 was confirmed by Western blot. Briefly, electrophoresed whole cell lysate samples were transferred to nitrocellulose membranes. The membranes were incubated with a 1:10,000 dilution of anti-FLAG mouse monoclonal antibody followed by incubation with a 1:5,000 dilution of a goat anti-mouse IgG monoclonal antibody conjugated to Alexa Fluor 680 (Life Technologies). Images were acquired using an Odyssey infrared imaging system (LI-COR Biosciences).

\section{RESULTS}

\section{Only the First of the 11 Sequential Clonal Variants of B. cenocepacia Produce OAg}

Chronically infecting bacteria in CF lungs often display changes in their LPS OAg (Maldonado et al., 2016). Therefore, we investigated the electrophoretic profiles of LPS extracted from the series of 11 sequential clonal variants of $B$. cenocepacia. SDS-PAGE followed by silver staining revealed that OAg was only present in the early isolate (IST439), while all other variants produce only lipid A-core devoid of OAg (Figure 1). The banding pattern of the OAg in IST439 was distinct from that in the strain K56-2 (Supplementary Figure S1). Therefore, although both IST439 and K56-2 belong to the same B. cenocepacia clonal group $^{2}$ they produce seemingly different OAg molecules.

\section{Chemical Structure of the LPS of $B$. cenocepacia Isolates with or without O-Antigen}

We investigated the chemical structure of the LPS produced by $B$. cenocepacia IST439 and also the structure of three randomly selected subsequent isolates that lacked OAg. Monosaccharide compositional analysis of the LPS from variants IST4113, IST4129 and IST4134 (Table 1) gave identical results (Supplementary Table S3), which also agreed with previously reported data for B. cenocepacia J2315 (Silipo et al., 2007). In contrast, the IST439 LPS had D-ribose and D-galactosamine

${ }^{2} \mathrm{http}: / /$ pubmlst.org/bcc residues as the main constituents. Results of linkage analyses of LPS from variants IST4113, IST4129, and IST4134 was also identical to that of J2315 (Silipo et al., 2007), but data obtained from IST439 LPS indicated also the presence of 2 -substituted ribofuranose and 4-substituted galactosamine. The fatty acids content of the LPS in the four IST strains also matched the archetypal Burkholderia lipid A components, namely $(R)$-3-hydroxyhexadecanoic acid [C16:0 (3-OH)] in amide linkage, and $(R)$-3-hydroxytetradecanoic [C14:0 (3-OH)] and tetradecanoic acid (C14:0) in ester linkage (Silipo et al., 2007).

Mild acidic conditions were used to characterize the monosaccharide sequence of the carbohydrate fraction of the purified LPS samples. This process cleaves the labile glycosidic linkage between the saccharide and the lipid A moieties. The carbohydrate fractions were purified by gel-permeation chromatography and analyzed by 1D and 2D NMR spectroscopy. The overlapped ${ }^{1} \mathrm{H}$-NMR spectra relative to all the isolates carbohydrate fractions were reported in Figure 2. It was immediately evident that the ${ }^{1} \mathrm{H}$ NMR spectra of IST4113, IST4129, and IST4134 were identical, highlighting the presence of eight anomeric signals relative to the sugar units composing the core moieties. In contrast, the IST $439{ }^{1} \mathrm{H}$ NMR spectrum showed two main signals in the anomeric region (Figure 2 and Table 2), attributed to the OAg domain. The complete LPS core oligosaccharide structures of isolates IST4113, IST4129, IST4134, and IST439 was assigned by the combination of data obtained from chemical analyses and 2D NMR spectroscopy using DQF-COSY, TOCSY, NOESY, ROESY, ${ }^{1} \mathrm{H},{ }^{13} \mathrm{C}-\mathrm{HSQC}$, and ${ }^{1} \mathrm{H},{ }^{13} \mathrm{C}$-HMBC. In all of these cases, the core was structurally identical to that previously described for B. cenocepacia J2315 (Silipo et al., 2007) except that the IST samples lacked the rhamnose- $N$-acetyl quinovosamine (Rha-QuiNAc) disaccharide linked to the outer core. In J2315, this disaccharide corresponds to a truncated component of the OAg (Ortega et al., 2009). Figure $3 \mathrm{~A}$ shows the core structure, elucidated by NMR spectroscopy, of isolates IST4113, IST4129, IST4134.

As stated above, NMR spectroscopy of the carbohydrate fraction from isolate IST439 identified two spin systems in the ${ }^{1} \mathrm{H}$-NMR spectrum, belonging to the $\mathrm{OAg}$, designed as $\mathbf{A}(\mathrm{H}-1$ at $\delta=5.09 \mathrm{ppm})$ and $\mathbf{B}(\delta=4.99 \mathrm{ppm})$ (Figure 2). Spin system $\mathbf{A}$ was identified as ribofuranose due to the correlations observed in the DQF-COSY and TOCSY spectra from the $\mathrm{H}-1$ A signal up to the diastereotopic methylene signal at position $\mathrm{H}-5$. The furanose form was established due to the presence of the typical low field shifted ring carbon atom signals (Figure $\mathbf{4}$ and Table 2). The anomeric configuration was attributed on the basis of the low ${ }^{3} J_{\mathrm{H}-1, \mathrm{H}-2}$ value attained from the DQF-COSY spectrum, indicative of a $\beta$-configuration in aldofuranose rings (less than $2 \mathrm{~Hz}$ ), and by the chemical shift of C-4 (around $83.0 \mathrm{ppm}$ in case of $\beta$-configuration) (Ahrazem et al., 2002, 2007). The downfield displacement of C-2 signal (Figure 4) indicated glycosylation at this position. Spin system B (H-1 at $\delta=4.99 \mathrm{ppm})$ was identified as an $\alpha$-galacto- configured monosaccharide, as attested by the low ${ }^{3} J_{\mathrm{H} 3, \mathrm{H} 4}$ and ${ }^{3} J_{\mathrm{H} 4, \mathrm{H} 5}$ values ( 3 and $1 \mathrm{~Hz}$, respectively), and the TOCSY correlations between the anomeric signal and the other ring protons up to the $\mathrm{H}-4$ resonance. Moreover, the 


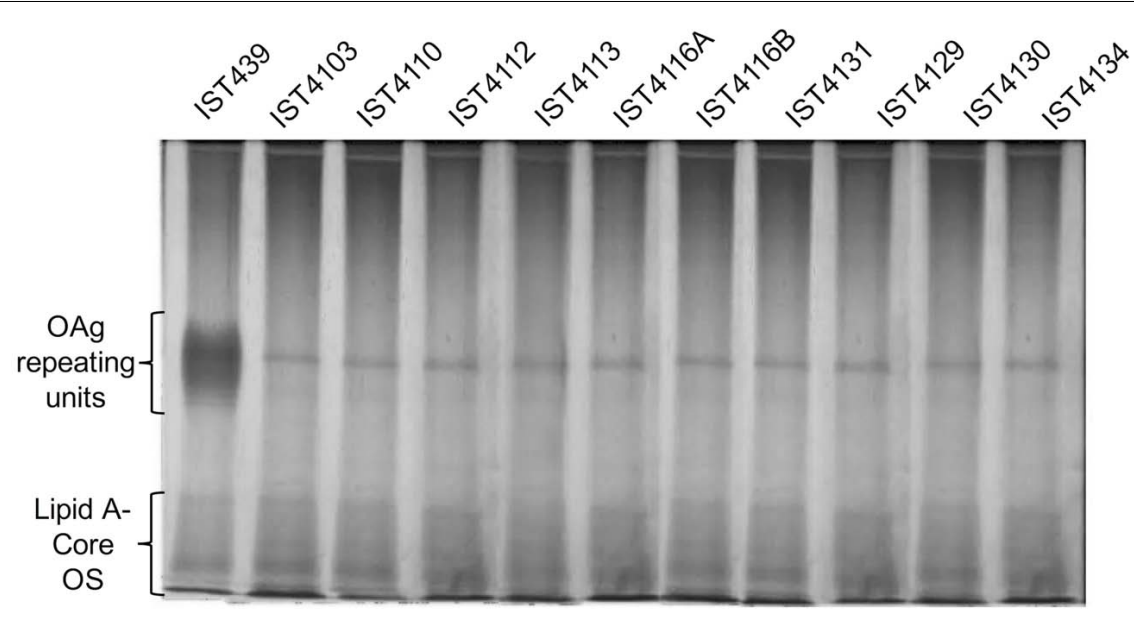

FIGURE 1 | Electrophoretic profile of the LPS extracted from 11 clonal Burkholderia cenocepacia (recA lineage IIIA) isolates retrieved from a chronically infected CF patient (in chrononological order). LPS loading was standardized based on culture optical density. Samples were run in $14 \%$ polyacrylamide gels (at $150 \mathrm{~V}$ for about $1 \mathrm{~h} 40 \mathrm{~m}$ ) in a Tricine-SDS system and developed by silver staining. The band that appears present in all the gels in the zone of the O-antigen (OAg) repeating units corresponds to residual proteinase $\mathrm{K}$.

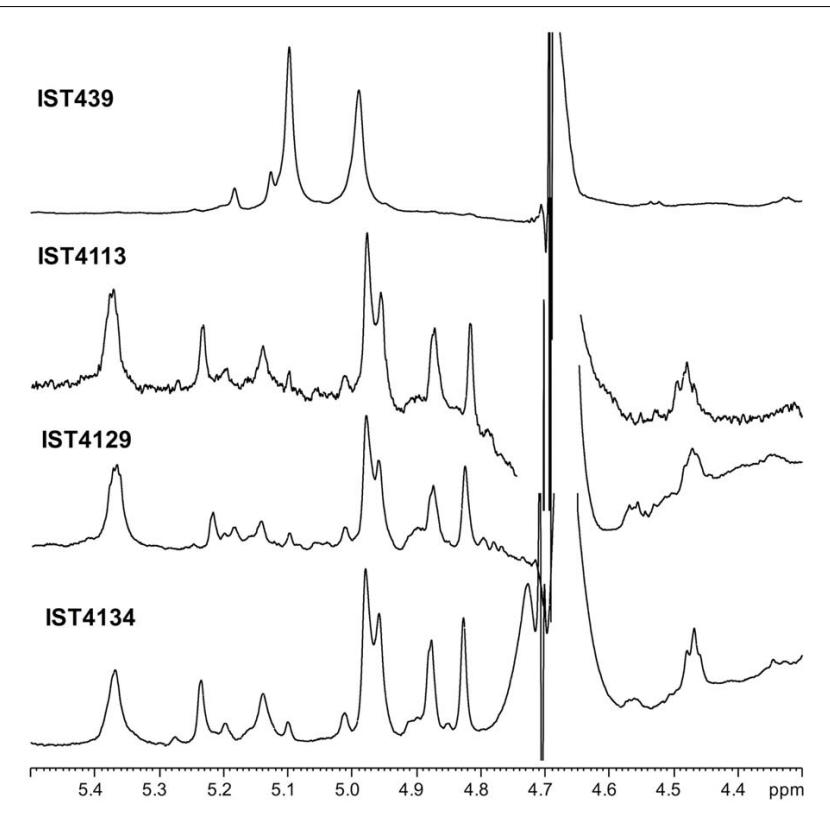

FIGURE $2 \mid{ }^{1} \mathrm{H}$ NMR spectrum of products obtained from IST439 and other three subsequent variants after mild acid hydrolysis. anomeric proton and carbon chemical shifts $(\delta=4.99 \mathrm{ppm}$ and $95.5 \mathrm{ppm})$, the intra-residue NOE correlation of $\mathrm{H}-1$ B with $\mathrm{H}-2 \mathbf{B}$, and the ${ }^{3} \mathrm{~J}_{\mathrm{H} 1, \mathrm{H} 2}$ were all in agreement with an $\alpha$-anomeric configuration and a ${ }^{4} C_{1}$ ring conformation. The detection of a signal for C-2 at 50.0 ppm (Figure 4) indicated that C-2 was a nitrogen-bearing carbon atom. Moreover, the downfield shift of proton resonance of $\mathrm{H}-2 \mathbf{B}$ was indicative of $\mathrm{N}$-acetylation at this position, as also corroborated by the NOE contact of H-2 B with the methyl protons of the acetyl group resonating at $\delta=1.92 \mathrm{ppm}$. Therefore, residue $\mathbf{B}$ was identified as a $\alpha$-GalNAc. The downfield shift of its $C-4(\delta=78.9 \mathrm{ppm})$ was indicative of glycosylation at this position. ROESY and NOESY spectra (not shown) allowed to detect the dipolar correlations necessary to assign the primary sequence of the IST439 LPS OAg repeating unit. The anomeric proton of GalNAc $\mathbf{B}$ gave a NOE correlation with $\mathrm{H}-2$ signal of ribose $\mathbf{A}$, whose anomeric signal showed a NOE correlation with the $\mathrm{H}-4$ of residue $\mathbf{B}$. These results, confirmed by the long scalar range connectivity derived from the ${ }^{1} \mathrm{H},{ }^{13} \mathrm{C}$-HMBC spectrum (not shown), demonstrated that the IST439 OAg structure consisted of a repeating disaccharide of $\beta$-D-ribofuranose $(\beta$-D-Ribf $)$ and $\alpha$-D-N-acetylgalactosamine ( $\alpha-\mathrm{D}$-Gal $p N A c$; Figure $3 \mathrm{~B})$. This structure is similar to that previously identified in $B$. cepacia (formerly Pseudomonas cepacia) serogroups O3 and O5 LPS OAg structures (Cox and Wilkinson, 1989).

Negative-ion MALDI mass spectrometry (MS) of the intact IST439 LPS showed ion peaks corresponding to lipid A and core species, which arose from the cleavage of the labile glycosidic linkage between Kdo and lipid A. Ion peaks corresponding to lipid A species were assigned as previously described (Silipo et al., 2007). The ion peak OS1 $(m / z=2033.3)$ agreed with an oligosaccharide composed of five heptoses, three hexoses, one Kdo, one D-glycero-D-talo-oct-2-ulosonic acid (Ko) and one 4-L-amino-4-deoxyarabinose (Ara4N), while ion peak OS2

TABLE 2 | Chemical shift $\delta\left({ }^{1} \mathrm{H} /{ }^{13} \mathrm{C}\right)$ of the O-chain moiety from B. cenocepacia IST439.

\begin{tabular}{lcccccc}
\hline \multicolumn{7}{c}{ Chemical shift $\delta\left(\boldsymbol{H}^{\mathbf{1 3}} \boldsymbol{C}\right)$} \\
\hline Unit & $\mathbf{1}$ & $\mathbf{2}$ & $\mathbf{3}$ & $\mathbf{4}$ & $\mathbf{5}$ & $\mathbf{6}$ \\
\hline A & 5.09 & 4.15 & 4.03 & 3.95 & $3.77-3.59$ & - \\
2- $\beta$-Rib & 106.6 & 78.7 & 67.2 & 82.3 & 62.5 & - \\
B & 4.99 & 4.02 & 3.93 & 3.61 & 3.63 & 4.03 \\
4- $\alpha$-GalNAc & 95.5 & 50.0 & 76.2 & 78.9 & 60.5 & 70.8
\end{tabular}




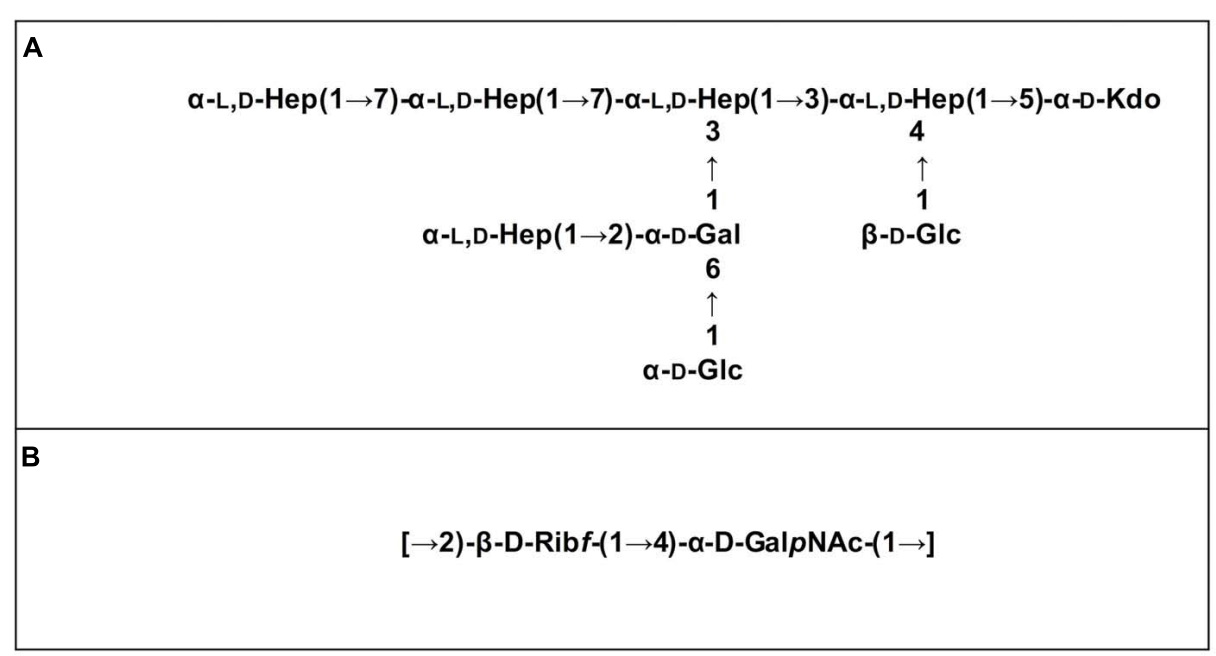

FIGURE 3 | LPS moieties structures elucidated by NMR spectroscopy. Core structure of isolates IST439, IST4113, IST4129, and IST4134 (A) and OAg repeating disaccharide of isolate IST439 (B)

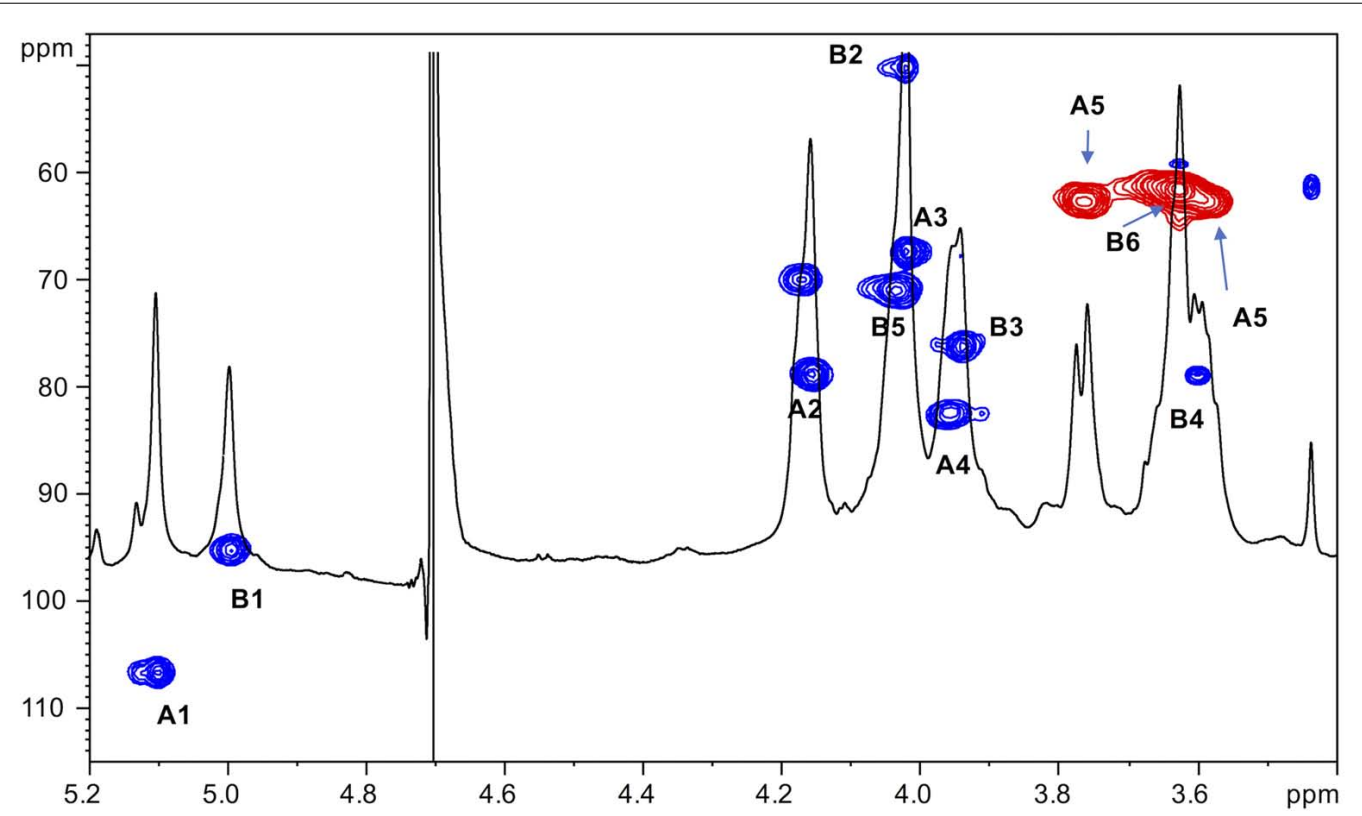

FIGURE $4 \mid{ }^{1} \mathrm{H},{ }^{13} \mathrm{C}-\mathrm{HSQC}$ spectrum of the O-chain moiety from IST439 strain. The heteronuclear correlations are indicated.

$(m / z=2166.2)$ corresponded to the same oligosaccharide carrying an additional Ara4N residue (Figure 5). Thus, the ion peaks derived from the IST439 core agreed with the structure previously elucidated (Silipo et al., 2007). The mass range 3000-4000 Da (Figure 5), containing peaks relative to either the oligosaccharide and the lipid A species, also confirmed previous studies (Silipo et al., 2007). Likewise, negative-ion MALDI mass spectra executed on the intact LPS isolated from the IST4113, IST4129, and IST4134 variants were identical each other and to the previously described mass spectra performed on intact B. cenocepacia ET-12 strain J2315 LPS
(Ortega et al., 2009). The MALDI mass spectrum of the intact IST4113 LPS, presented in Supplementary Figure S2, showed at mass range $m / z 1300-2500 \mathrm{Da}$, OS species composed of four to six heptoses and three to five hexoses, one Kdo, one Ko, and with or without Ara4N. In particular, the ion peak termed OS $(m / z=2032.5$, Supplementary Figure S2) was indicative of the core previously elucidated (Silipo et al., 2007). The LPS molecular ions in the mass range 3000-4200 Da (Supplementary Figure S2) also confirmed previously reported data and agreed with chemical and NMR spectroscopy analyses. 


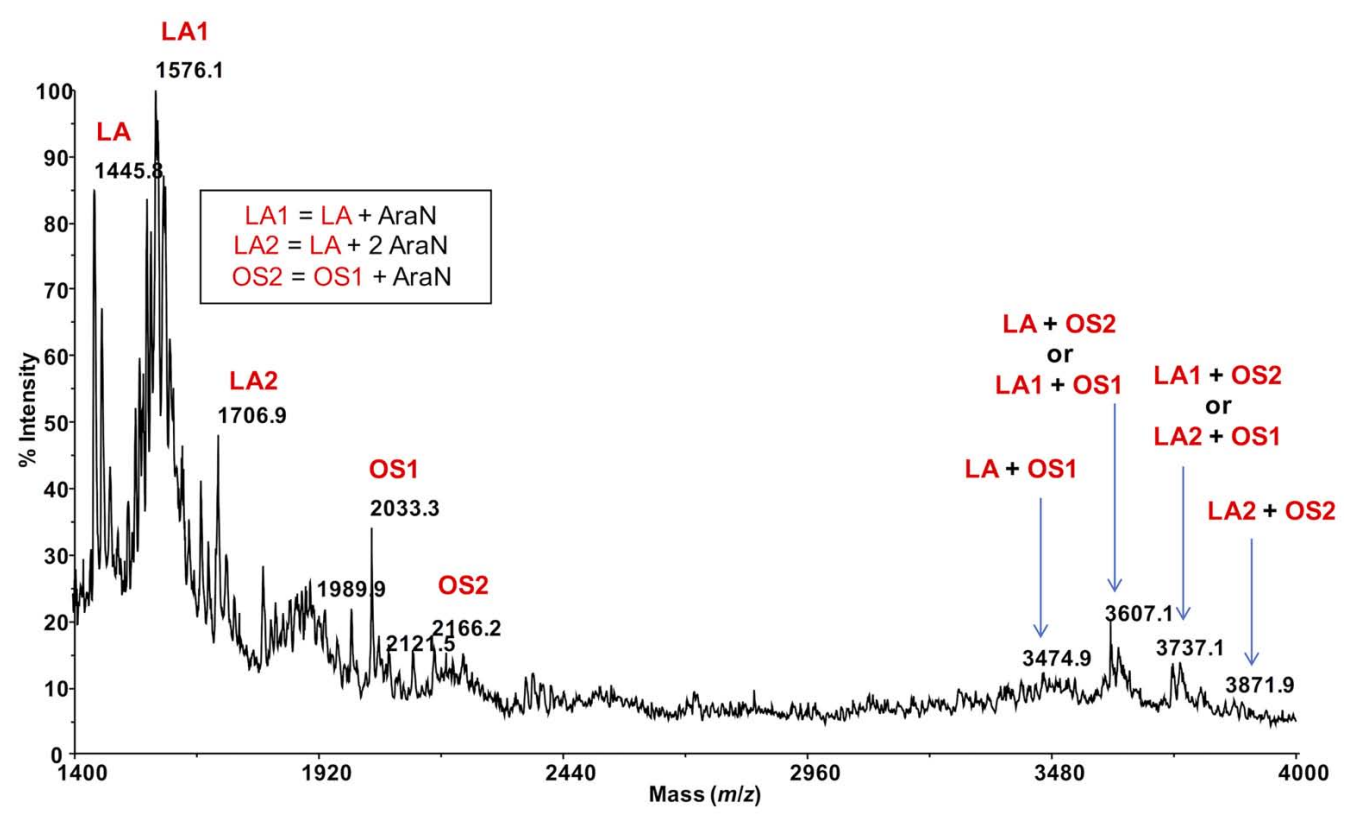

FIGURE 5 | MALDI mass spectrum of the intact LPS from strain IST439 (mass range 1400-4000). Lipid A-core oligosaccharide molecular ions and their ion fragments, attributable to the core OS and to the reported lipid A structure(s), are indicated.

\section{Genomic Sequence of the B. cenocepacia Clonal Isolates Reveals of a Novel Hybrid O-Antigen Biosynthetic Cluster}

The $11 B$. cenocepacia sequential isolates studied here belong to the recA-lineage IIIA/ET-12, as the prototypic epidemic strains K56-2 and J2315 (Coutinho et al., 2011a; Mira et al., 2011). The genome sequences of the 11 isolates were used to investigate the genetic organization of the OAg biosynthetic gene cluster in comparison to that of B. cenocepacia K56-2 (Ortega et al., 2005) (Figure 6). The cenocepacia K56-2 OAg biosynthetic cluster is on chromosome 1 and spans approximately $29 \mathrm{~kb}$ comprising 24 genes (Figure 6) flanked by ure $G$ and $a p a H$ (Ortega et al., 2005). The B. cenocepacia IST439 OAg biosynthetic cluster is also located in chromosome 1 , flanked by $u r e G$ and $a p a H$, and spans a region of $29.5 \mathrm{~kb}$. The cluster consists of 23 ORFs (Figure 6), most of them functionally assigned based on the bioinformatic analysis of predicted polypeptides. Like B. cenocepacia K56-2, the IST439 OAg cluster does not have a transposon insertion element inserted within the cluster (Supplementary Figure S3) (Ortega et al., 2005). Sixteen of the 23 ORFs in IST439 are homologs to B. cenocepacia K56-2/J2315 genes, including waaA, wbxY (kdoO), waaC, manB, wzx, wbxA, wbxB, gale, wecA, wbiI, wbiH, wbiG, $r m l D, r m l C, r m l A$, and $r m l B$. However, the remaining seven genes (wbiF, bmul_2508, bmul_2509, bmul_2510, bmul_2514, $w z t$, and $w z m)$ are closer in terms of sequence similarity to ORFs in B. multivorans ATCC 17616 [environmental isolate with a complete genome deposited at NCBI and Bcc databases (Nishiyama et al., 2010)], ranging from 79 to $96 \%$ of amino acid identity, versus no homology (at an expect value cut-off of $1 \mathrm{E}-4)$ with any known B. cenocepacia strains for bmul_2508, bmul_2509, bmul_2510, and amino acid identity of 31 to $74 \%$ with the closely related ET-12 B. cenocepacia J2315 for the remaining four genes. This observation suggested a hybrid origin of this OAg cluster in IST439 (Supplementary Figures S2, S3). Analysis of the GC content across the IST439 OAg cluster shows a sharp drop in the region containing the seven genes related to B. multivorans (average content $56.1 \%$ in B. cenocepacia IST439 and $55.3 \%$ in B. multivorans ATCC 17616; Figures 6A, 7B, respectively) in comparison to the rest of the cluster (average content $63.1 \%$, and $66.6 \%$ when subtracting the seven genes) and the IST439 complete genome (average content 67.4\%). This variation in GC content is usually found in genomic islands of prokaryotic genomes and is a hallmark of regions undergoing frequent recombination, as is the case of the LPS synthetic locus (Zhang et al., 2014; Maldonado et al., 2016).

The waaA, kdoO, waaC, and manB genes (represented in gray in Figure 6) encode proteins involved in lipid A-core biosynthesis (Ortega et al., 2005). In particular, the predicted amino acid sequence of $\mathrm{WaaA}$ and $\mathrm{WaaC}$ are highly identical to 3-deoxy-D-manno-octulosonic acid and heptosyltransferase I, respectively, and they are therefore involved in inner core LPS synthesis. $w b x Y$ (BCAL3311), previously annotated as a gene encoding a conserved protein of unknown function (Ortega et al., 2005), encodes a Kdo dioxygenase that is responsible for the conversion of the distal Kdo residue of the Burkholderia lipid A-core into Ko (Chung and Raetz, 2011; Chung et al., 2014; Fathy Mohamed et al., 2017), and it was renamed as $k d o O$. Another conserved functional feature in this OAg cluster is the predicted transmembrane protein encoded by wbiH, which includes functional features of the wecA family (Valvano, 2003; 


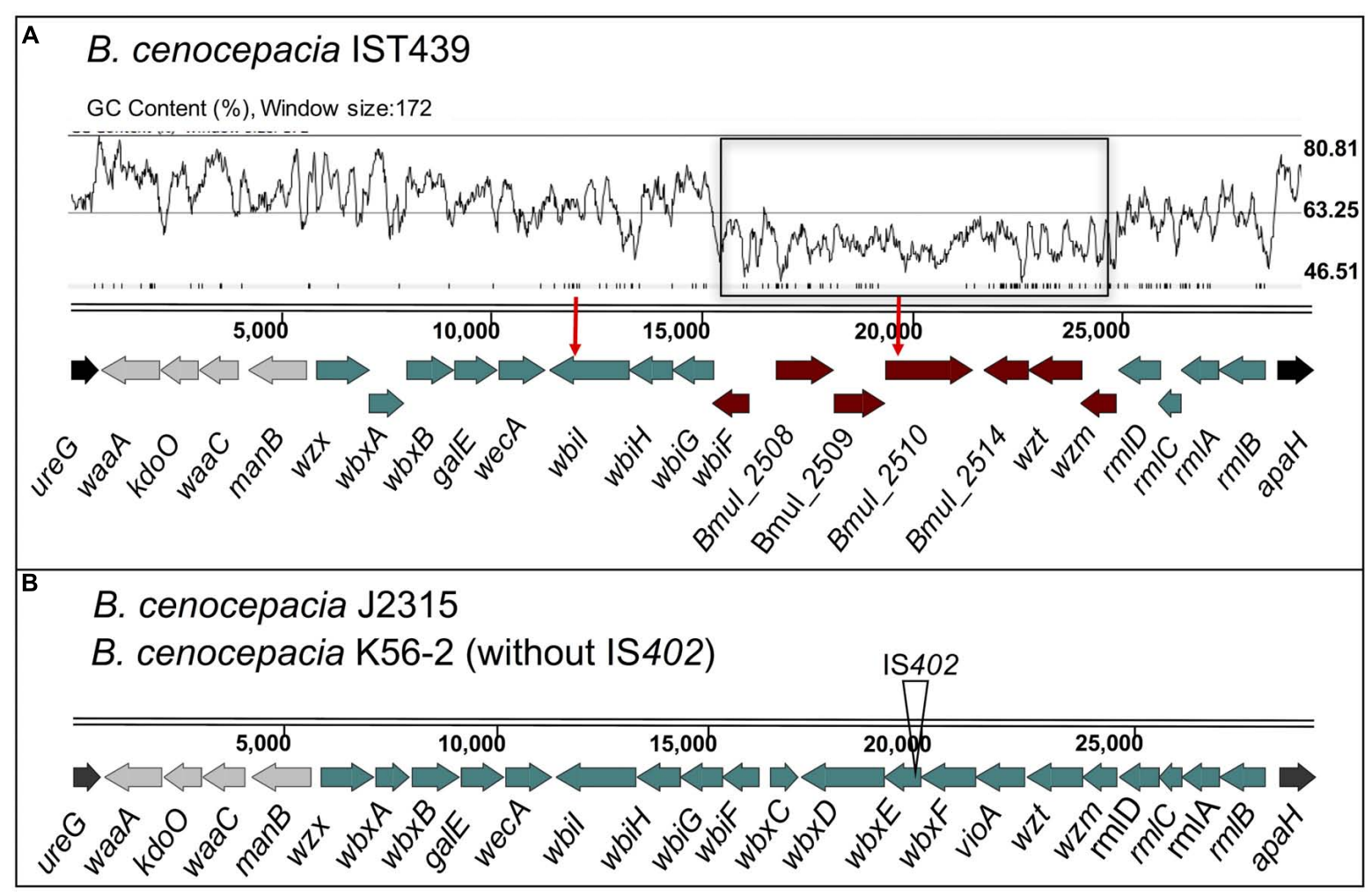

FIGURE 6 | Genetic organization of gene clusters for core-lipid A and OAg biosynthesis in B. cenocepacia IST439 (A) and the reference strains K56-2 and J2315 (B). The flanking genes ure $\mathrm{G}$ and $\mathrm{apaH}$ are indicated in black and the four genes represented in gray encode proteins putatively involved in lipid A-core biosynthesis. The genes in dark-red correspond to genes present in IST439 without a counterpart in the reference strain J2315 (Supplementary Figure S3), but with a degree of homology to B. multivorans ATCC 17616 (see also Supplementary Figure S4B). The red-vertical arrows above the genes represent the two non-synonymous point mutations in genes bmul_2510 and wbil of the 10 sequential isolates (comparative genomic analysis to be described elsewhere). A GC content plot is also represented for IST439 [drawn using Artemis (Carver et al., 2008)] above the display line of the sequence, where the genes annotated as B. multivorans are highlighted in a black rectangle. waaA, 3-deoxy-D-manno-octulosonic acid transferase; kdoO, Kdo dioxygenase; waaC, heptosyltransferase I; manB, phosphomannomutase; $w z x, \mathrm{OAg}$ exporter; $w b x A$, glycosyltransferase; $w b x B$, glycosyltransferase; galE, UDP-glucose epimerase; wecA, UDP-N-acetylglucosamine 1-P transferase; wbil, nucleotide sugar epimerase-dehydratase; wbiH, UDP-N-acetylglucosamine 1-P transferase; wbiG, nucleotide sugar epimerase-dehydratase; wbiF, glycosyltransferase; bmul_2508, conserved hypothetical protein; Bmul_2509, group 1 glycosyl transferase; Bmul_2510, conserved hypothetical protein; Bmul_2514, type 11 methyltransferase; wzt, ABC transporter ATP-binding protein; wzm, ABC transporter membrane permease; rmIDCAB, dTDP-rhamnose biosynthesis; $w b x C$, acetyltransferase; $w b x D$, glycosyltransferase; $w b x E$, glycosyltransferase; vioA, nucleotide sugar aminotransferase.

Ortega et al., 2005). The wecA family proteins and other proteins, encoded by galE, wbiI, and $w b i G$, are probably involved in the transfer of $\mathrm{N}$-acetylhexosamines to the undecaprenol-phosphate intermediate to initiate the assembly of the OAg subunits (Raetz and Whitfield, 2002; Valvano, 2003, 2015), while genes $w b x A$, $w b x B, w b i F$ and bmul_2509 encode glycosyltransferases likely involved in OAg elongation. Genes bmul_2508 and bmul_2510 encode proteins of unknown function, while bmul_2514 encodes a type-11 methyltransferase, possibly involved in the termination of OAg assembly. Genes wzm and wzt encode a two-component $\mathrm{ABC}$ transporter involved in OAg export across the cytoplasmic membrane (ABC-transporter-dependent pathway) after OAg polymerization on the cytoplasmic side (Raetz and Whitfield, 2002; Valvano, 2003, 2015). There is an alternative pathway of OAg export, the $w z y$-dependent pathway based on the concerted action of proteins Wzx and Wzy (Raetz and Whitfield, 2002; Valvano, 2003, 2015), but the OAg biosynthetic cluster of IST439 does not encode a homolog of $w z y$. This gene is also lacking in B. cenocepacia K56-2 (Ortega et al., 2005). Together, these observations indicate that the IST439 OAg, like in strain K56-2, is exported by an $\mathrm{ABC}$ transporter dependent pathway.

\section{Mutations in wbil and bmul_2510 Are Not Involved in Loss of OAg}

Whole-genome comparison of the $10 \mathrm{~B}$. cenocepacia clonal variants against IST439 identified only three mutational events spanning the entire OAg cluster, corresponding to three non-synonymous SNPs in coding regions IST439_01746 (homolog to B. cenocepacia bmul_2510) and wbiI (Figure 6A, red arrows). The point mutation in bmul_2510 is conserved in all sequential isolates and results in the replacement of a threonine for a proline residue (T116P) in the corresponding Bmul_2510 polypeptide. In contrast, wbiI has two different point mutations resulting in L493P replacement in three isolates (IST4112, IST4113, and IST4116B) and E489K in other six isolates (IST4110, IST4116A, IST4131, IST4129, IST4130, and 


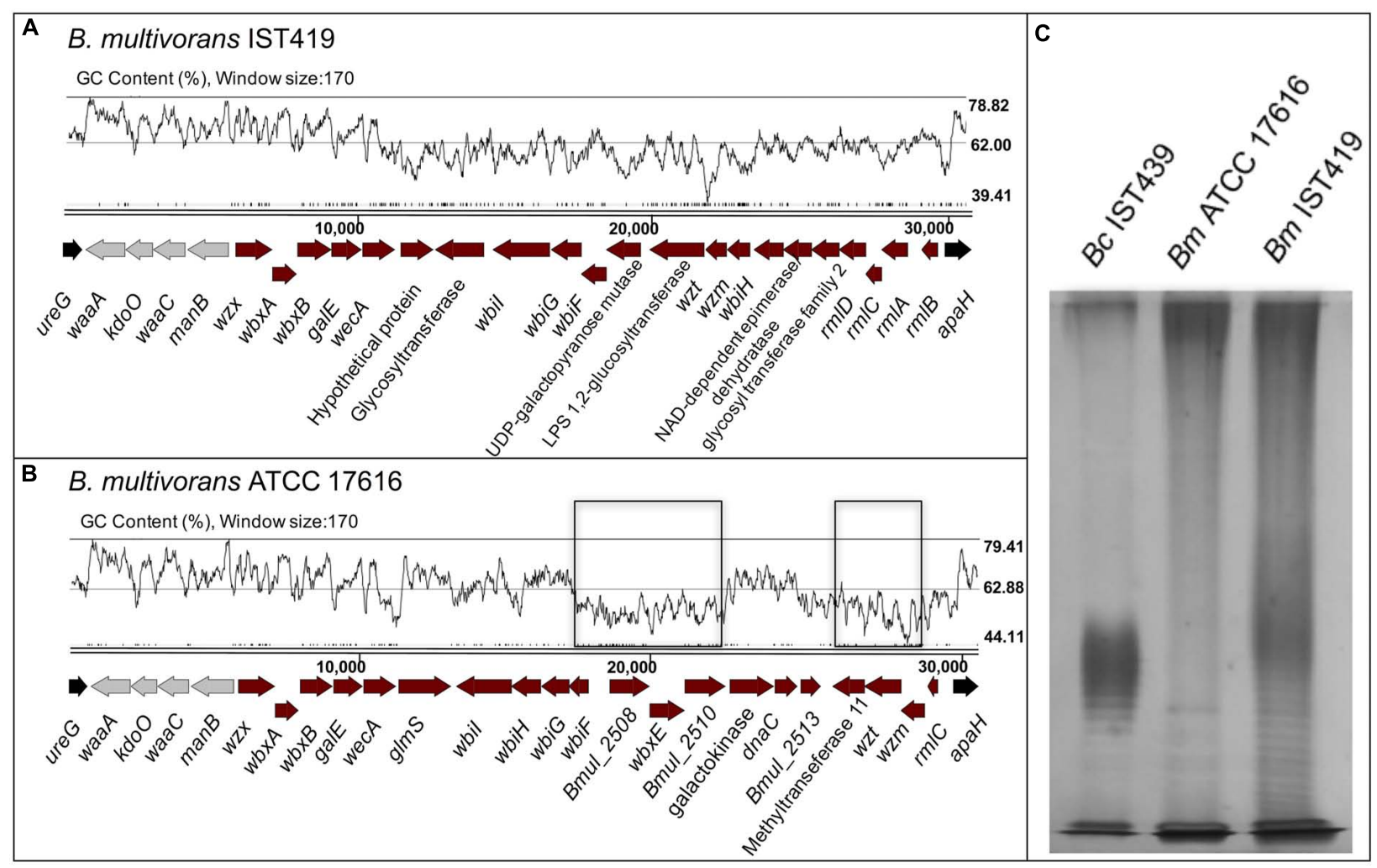

FIGURE 7 | Genetic organization of the OAg biosynthetic gene clusters of B. multivorans strains ATCC 17616 (B) and IST419 (A), including genes for lipid A-core biosynthesis and OAg biosynthesis within the apaH and ureG flanking genes (in black). Conserved genes among Burkholderia species involved in lipid A-core biosynthesis are indicated in gray. GC content plots are represented for both clusters [drawn using Artemis (Carver et al., 2008)], where the B. multivorans ATCC 17616 with homology in the corresponding cluster of B. cenocepacia IST439 are highlighted in a black rectangle. A silver nitrate-stained SDS-PAGE gel (C) shows the banding pattern of LPS samples extracted from B. cenocepacia IST439 (BC IST439), B. multivorans IST419 (Bm IST419), and B. multivorans ATCC 17616 (Bm ATCC17616).

IST4134). No other mutations were identified within the OAg biosynthetic locus. Further, no mutations were found in the OAg ligase waaL and in the wabO-dnaE cluster that contains the reminder of the core-oligosaccharide biosynthesis genes (Ortega et al., 2009). However, eleven conserved SNPs were found outside the OAg cluster as well as one mutational event - as a deletion - in the late variants, compared with IST439, but none of them appear to be directly related to the loss of the OAg. Since the bmul_2510 mutation is in all isolates after IST439, and wbiI mutations are present in nine isolates (except for IST4103), we performed complementation experiments in IST4103 with the bmul_2510 homolog gene cloned from IST439 as an attempt to reconstitute OAg biosynthesis (Cardona and Valvano, 2005). The bmul_2510 coding sequence including a FLAG tag epitope was placed under the control of a rhamnoseinducible promoter and the complemented strain was grown at various rhamnose concentrations. However, we could not detect OAg production in IST4103 (Figure 8). We also attempted complementation experiments for bmul_2510 and wbiI singly or in combination using strain IST4134, but none of these experiments resulted in restoration of OAg synthesis (Figure 8). Failure to complement OAg synthesis was not due to lack of protein expression since bmul_2510and wbil-encoded polypeptides containing FLAG tags were detectable by Western blot analysis (Figure 8B). Additional bands reactive against the FLAG monoclonal antibodies correspond to cross-reactive proteins, as they also appear in the control lanes of lysates from bacteria containing empty vector. Therefore, from these results we conclude that the gene polymorphisms in the OAg biosynthesis cluster cannot explain the loss of OAg production in the sequential isolates obtained after IST439.

\section{Analysis of the OAg Cluster of a Co-infecting B. multivorans Isolate Rules Out that the Hybrid Cluster in IST439 Arose from Gene Transfer in the Lung}

Because of the hybrid nature of the IST439 OAg biosynthetic cluster and the isolation of co-infecting B. multivorans in the same patient, we investigated the possibility that this cluster could have arisen from a gene transfer event in patient. B. multivorans IST419 was collected approximately 1 year before B. cenocepacia IST439 in patient J (Table 1) (Cunha et al., 2003). The combination of two sequencing rounds of $B$. multivorans 


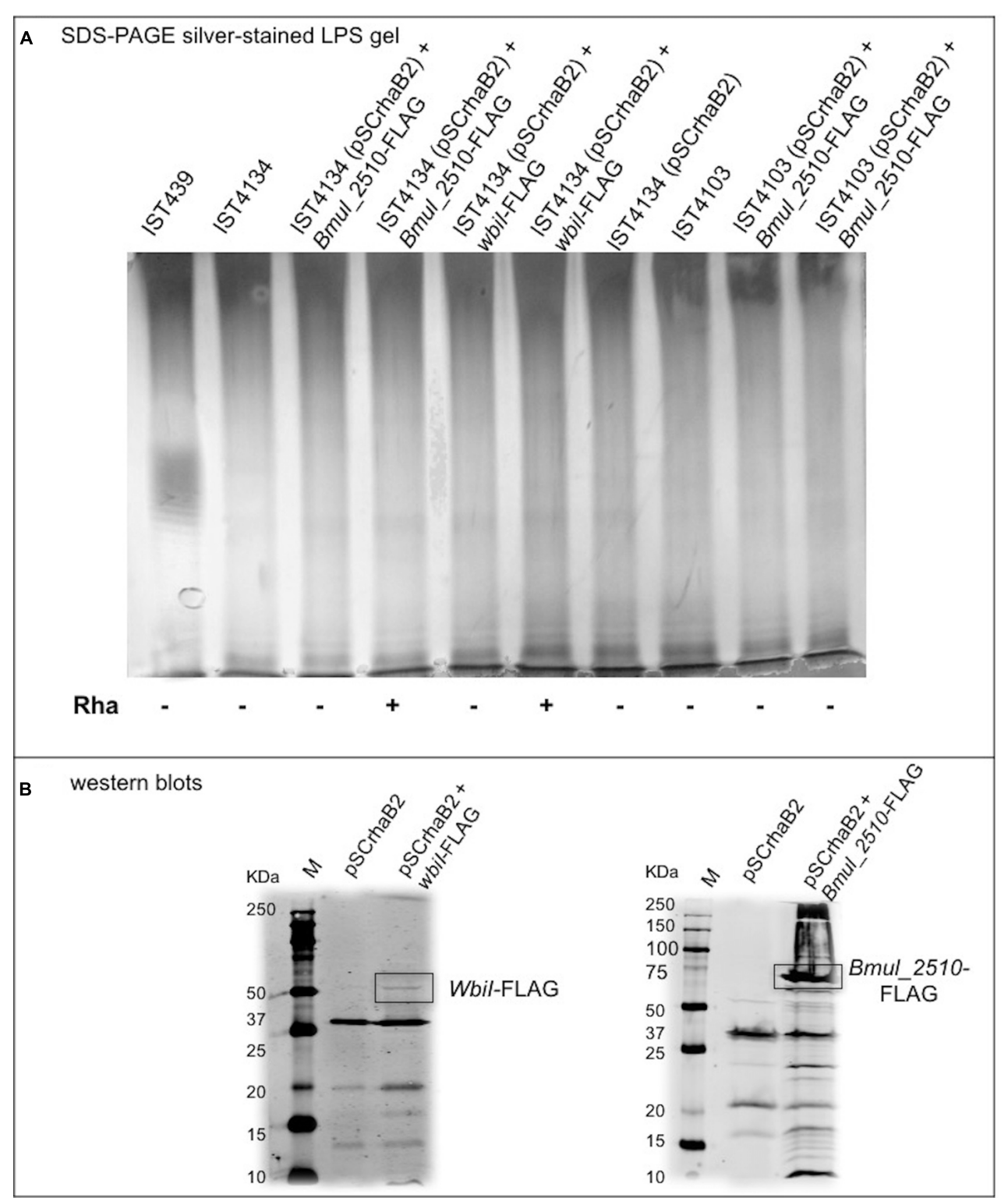

FIGURE 8 | Characterization of the OAg unit after cloning and introduction of B. cenocepacia IST439 wbil and Bmul_2510 genes in all variants lacking OAg production to complement the corresponding mutated genotypes. (A) LPS electrophoretic profiles for IST439, IST4134, and IST4103 showing the presence/absence of OAg units, and OAg absence in all transconjugants selected; (+) denotes $1 \%$ (w/v) rhamnose and $(-)$ denotes no rhamnose added to the medium. (B) Western blots showing bands at around $60 \mathrm{KDa}$ (left) and $75 \mathrm{KDa}$ (right), which indicate Wbil and Bmul_2510 polypeptides, respectively. Lanes shown (left to right): protein ladder (M), cloning vector pSCrhaB2, cloning vector + Wbil-FLAG or Bmul_2510-FLAG.

IST419 allowed us to assemble 155 contigs (N50 $=171331)$ with a predicted genome size of $\sim 6.5 \mathrm{Mb}$ and 5818 genes (Zerbino and Birney, 2008; Seemann, 2014). Using this annotated assembly, the corresponding OAg genetic cluster was identified and compared to that of B. multivorans ATCC 17616 (Nishiyama et al., 2010), B. multivorans D2095 (NCBI nucleotide accession number: JFHP00000000.1) (Silva et al., 2016), and B. cenocepacia IST439. The OAg biosynthetic cluster in B. multivorans IST419, also located in chromosome 1, includes 25 ORFs spanning a $30.8 \mathrm{~kb}$ segment flanked by ureG and apaH (Figure 7A). Functional assignments could be made to most of the genes based on bioinformatics. This OAg cluster has more ORFs than IST439 but shows three conserved features: first, the region involved in lipid A-core biosynthesis (waaA, kdoO, waaC, and $\operatorname{man} B)$, second, the presence of $w b i H$ and $w e c A$ encoding the putative initiating enzyme for OAg biosynthesis, and third, the presence of conserved genes $r m l B A C D$ encoding enzymes for dTDP-rhamnose synthesis (Valvano, 2003, 2015; Ortega et al., 2005). A detailed analysis of this cluster, together with the alignment of filtered fastq files of $B$. multivorans IST419 against the same region of $B$. multivorans ATCC 17616 (Figure 7B and Supplementary Figure S4C), shows that B. multivorans-like genes 
in the IST439 OAg cluster are not present in B. multivorans IST419 (Supplementary Figure S4A). Further, a similar analysis using a second clinical B. multivorans isolate as reference (B. multivorans D2095 (Silva et al., 2016)), also revealed the complete absence of homologs to those specific B. multivorans ATCC 17616 genes. The electrophoretic analysis of LPS samples obtained from B. cenocepacia IST439, B. multivorans IST419, and B. multivorans ATCC 17616 showed that the first two express an OAg possessing repetitive units with different banding patterns, but B. multivorans ATCC 17616 does not produce an OAg unit (Figure 7C). Therefore, we conclude that in vivo gene transfer over the course of the infection of the patient can't explain the hybrid gene organization of the OAg cluster in IST439 and related serial isolates.

To shed more light on the origin of the hybrid OAg biosynthetic cluster in B. cenocepacia IST439, we determined the genetic organization of the corresponding region in the environmental isolate B. multivorans ATCC 17616 (Figure 7B). The gene cluster spans a region of $31.4 \mathrm{~kb}$ in chromosome 1 containing 24 predicted ORFs. In terms of functional conservation, the B. multivorans ATCC 17616 region is similarly organized to that of B. cenocepacia IST439 (Supplementary Figure S4B) although, unlike B. cenocepacia IST439, the seven genes annotated in B. multivorans ATCC 17616 are not consecutively organized in the $\mathrm{OAg}$ cluster (indicated by black boxes in Figures 6A, 7B).

\section{DISCUSSION}

Phenotypic diversity and genotypic flexibility of $B$. cenocepacia during long-term infection of CF lungs was recently well established using genomic and phenotypic analyses of serially collected clinical isolates (Lee et al., 2017). In this study, we characterized a novel hybrid genetic LPS OAg locus in the early $B$. cenocepacia isolate of a series of sequential isolates obtained over a 3.5-year period from a patient with CF. We also demonstrate that all of the 10-subsequent clonal $B$. cenocepacia variants collected from the same patient until shortly before death do not produce OAg (Figure 1). This observation provides further evidence for the OAg loss during $B$. cenocepacia adaptation over chronic lung infection (Maldonado et al., 2016).

We elucidated the chemical structure of the lipid A-core of four of the 11 isolates, as well as the structure of the OAg in the early isolate (IST439). The lipid A-core of these strains had the same structure as that described for other members of the Burkholderia genus (De Soyza et al., 2004, 2008). Indeed, the lipid A moiety shows the typical Burkholderia glucosamine disaccharide backbone with the $[\mathrm{P} \rightarrow 4-\beta-\mathrm{D}-\mathrm{Glc} p \mathrm{~N}-(1 \rightarrow 6)-\alpha-\mathrm{D}-$ Glc $p \mathrm{~N} 1 \rightarrow \mathrm{P}$ ] sequence that comprises two Ara4N residues linked to phosphate groups. The inner core contains the characteristic Ara4N-Ko-Kdo trisaccharide, previously elucidated in other Burkholderia species (Isshiki et al., 1998; Gronow et al., 2003), and is extended by a heptose-rich sequence. The OAg repeating unit of the LPS in IST439 was a disaccharide $[\rightarrow 2)-\beta$-D-Ribf$(1 \rightarrow 4)-\alpha-D-G a l p N A c-(1 \rightarrow]$, which was not previously described in B. cenocepacia, differs from the OAg structure of the prototypic strain K56-2 (Ortega et al., 2005, 2009), and is similar to that occurring in the $\mathrm{OAg}$ of $\mathrm{B}$. cepacia serotypes $\mathrm{O} 3$ and $\mathrm{O} 5$ (Cox and Wilkinson, 1989). In addition to the presence of a novel OAg, the absence of the Rha-QuiNAc disaccharide, linked to the $\alpha$-Hep- $(1 \rightarrow 2)-\alpha$-Gal disaccharide of the outer core in all the isolates investigated, represented a further novelty of these strains.

In keeping with the OAg structure, a new OAg biosynthetic locus was also discovered in this study. This genetic locus exhibits a hybrid origin, comprising genes with homology to B. multivorans ATCC 17616 and low to no amino acid similarity with other sequenced B. cenocepacia strains. Comparisons with the OAg locus of a co-infecting B. multivorans isolate (IST419), which was present at the time of acquisition of $B$. cenocepacia IST439 (Figure 7A) (Cunha et al., 2003), showed that the genetic organization of the $\mathrm{OAg}$ cluster in $B$. multivorans IST419 is different from that of B. cenocepacia IST439, with no homology with the B. multivorans-like genes shared by IST439 and environmental strain B. multivorans ATCC 17616 genomes. Together, our findings demonstrate that no transfer of genetic material occurred between the two co-infecting species in the lung of this CF patient during the infection within the OAg biosynthetic cluster.

The origin of the unique OAg locus of B. cenocepacia IST439 is unknown, but it was found that the $B$. multivorans-like region differs significantly from the GC content of the entire cluster and core genome (Figure 6A), which is a common indicator for the presence of a putative genetic island and former mobile element (Hacker and Carniel, 2001; Zhang et al., 2014). Studies mostly based on Salmonella enterica and E. coli (D'Souza et al., 2005; Hu et al., 2010; Reeves et al., 2013) have previously suggested that $\mathrm{OAg}$ biosynthetic operons in bacterial pathogens were acquired by horizontal genetic transfer from species with a low GC content. Moreover, horizontal exchange of O-specific antigen biosynthetic genes among phylogenetically distinct $P$. aeruginosa strains was observed and serotype switching was found to be the result of horizontal transfer and genetic recombination of LPS biosynthetic genes originating from an multidrug-resistant taxonomic outlier P. aeruginosa strain (Thrane et al., 2015). The high variation within the cluster also indicates that these $\mathrm{OAg}$ clusters might have been assembled by multiple transmission events over time (Lerouge and Vanderleyden, 2002; Thrane et al., 2015; Maldonado et al., 2016). In the case of B. cenocepacia IST439, the core genome and the OAg cluster (minus the 7-gene region) have a similar GC content (66-67\%), whereas the region containing the seven genes annotated as B. multivorans ATCC 17616, with a $56.1 \%$ GC content, is an obvious candidate for a mobile element that was acquired by horizontal genetic transfer and may be adaptive. However, the corresponding seven genes in B. multivorans ATCC 17616 are not encoded consecutively, being interrupted by a group of three high-GC content genes (Figure 7B), and cannot be matched to the same region in B. cenocepacia IST439 without assuming the occurrence of multiple mutational events. Based on these observations and the variability observed within the $\mathrm{OAg}$ cluster of the $B$. multivorans sequences examined, it can be suggested that this putative 
mobile element identified in the OAg biosynthetic cluster of B. cenocepacia IST439 has a foreign non-Bcc origin, which at some point might have also been acquired by an ancestor of B. multivorans ATCC 17616.

We could not establish a direct link between OAg loss in all 10 late-stage variants with the conserved mutation in the gene homolog to Bmul_2510 or with the mutations in wbiI since none of our complementation efforts using genetic constructions with B. cenocepacia IST439 Bmul_2510 and/or wbiI in different backgrounds could reconstitute OAg biosynthesis. Although it is possible that lack of complementation of OAg synthesis is associated with insufficient expression of the cloned gene or poor translocation of the protein to the membrane, it cannot be excluded that these mutations require the complementation of another genetic alteration in a distinct part of the genome with some role in OAg biosynthesis that remains to be identified. Alterations in the LPS molecule during chronic CF infections are thought to contribute to adhesion, evasion of immune defenses and overall adaptation to the infection niche (Maldonado et al., 2016). In $P$. aeruginosa, it is well established that adaptation to the lung during chronic infection includes the loss of OAg and/or lipid A modifications that allow the bacterium to avoid host immune responses (Lyczak et al., 2002; Cigana et al., 2009; Dettman et al., 2013; Maldonado et al., 2016). LPS virulence resides both in the endotoxin activities of the lipid $\mathrm{A}$ and in the ability of the core oligosaccharide and OAg to provide the bacterium with resistance to host defenses. The ability of 3 of the $11 \mathrm{~B}$. cenocepacia clonal variants to subvert host defenses was recently assessed using dendritic cells (Cabral et al., 2017), revealing that the late variants IST4113 and IST4134 were significantly more internalized than IST439, the only isolate that expresses the OAg unit, in line with previous studies by Saldias et al. (2009). Moreover, the late-stage isolates also exhibited improved survival within dendritic cells than the early isolate IST439, corroborating the idea that loss of the OAg may participate in providing an adaptive advantage to chronically infecting $B$. cenocepacia. Collectively, our results lend support to the notion that LPS OAg modifications are an important factor in the adaptation of $B$. cenocepacia to chronic infection and that that OAgs heterogeneity relates to variation within the OAg gene cluster.

\section{REFERENCES}

Ahrazem, O., Prieto, A., Leal, J., Jimenez-Barbero, J., and Bernabe, M. (2002). Fungal cell wall galactomannan isolated from Apodus deciduus. Carbohydr. Res. 337, 1503-1506. doi: 10.1016/S0008-6215(02) 00184-2

Ahrazem, O., Prieto, A., Leal, J. A., Inmaculada Gimenez-Abian, M., JimenezBarbero, J., and Bernabe, M. (2007). Fungal cell wall polysaccharides isolated from Discula destructiva spp. Carbohydr. Res. 342, 1138-1143. doi: 10.1016/j. carres.2007.02.011

Altschul, S. F., Gish, W., Miller, W., Myers, E. W., and Lipman, D. J. (1990). Basic local alignment search tool. J. Mol. Biol. 215, 403-410. doi: 10.1016/S00222836(05)80360- 2

\section{AUTHOR CONTRIBUTIONS}

FDL, AS, and AM were responsible for LPS-O-Ag structural analysis and CC and IS-C prepared Bcc cells for the analysis. RM was involved in the cloning and expression of $\mathrm{O}$-Ag genes in $\mathrm{MV}$ laboratory and $\mathrm{AH}, \mathrm{CC}$, and RM performed the electrophoretic profiles of LPS and the complementation assays. SdS, VC, and IS-C were involved in the genome sequencing and analysis of the B. cenocepacia clonal isolates and AH, SdS, RM, MV, $\mathrm{VC}$, and IS-C in the in silico analysis of Bcc O-Ag clusters. IS-C coordinated the work and the writing of the paper with contributions from the co-authors. All authors have read and approved the submission of this manuscript.

\section{FUNDING}

This research was funded by grants from $\mathrm{iBB}$ - Institute for Bioengineering and Biosciences from FCT - Portuguese Foundation for Science and Technology (UID/BIO/04565/2013) and from Programa Operacional Regional de Lisboa 2020 (Project No. 007317), FCT Ph.D. grants to AH (BIOTECnico - Ph.D. Program - PD/BD/113629/2015) and RM (SFRH/BD/84233/2012), and Post-Doctoral grants to CC (SFRH/BPD/81220/2011) and SdS (SFRH/BPD/75483/2010). RM's stay in MV's laboratory was supported by the EU COST Action BM1003.

\section{ACKNOWLEDGMENTS}

The collaborative work performed in IS-C, AM, AS, and MV laboratories was within the framework of the EU COST Action BM1003: Microbial cell surface determinants of virulence as targets for new therapeutics in cystic fibrosis (http://www.costbm1003.info/).

\section{SUPPLEMENTARY MATERIAL}

The Supplementary Material for this article can be found online at: http://journal.frontiersin.org/article/10.3389/fmicb. 2017.01027/full\#supplementary-material

Bolger, A. M., Lohse, M., and Usadel, B. (2014). Trimmomatic: a flexible trimmer for Illumina sequence data. Bioinformatics 30, 2114-2120. doi: 10.1093/bioinformatics/btu170

Cabral, M., Pereira, M., Silva, Z., Iria, I., Coutinho, C., Lopes, A., et al. (2017). Using dendritic cells to evaluate how Burkholderia cenocepacia clonal isolates from a chronically infected cystic fibrosis patient subvert immune functions. Med. Microbiol. Immunol. 206, 111-123. doi: 10.1007/s00430-016-0488-4

Cardona, S. T., Mueller, C. L., and Valvano, M. A. (2006). Identification of essential operons with a rhamnose-inducible promoter in Burkholderia cenocepacia. Appl. Environ. Microbiol. 72, 2547-2555. doi: 10.1128/AEM.72.4.2547-2555. 2006

Cardona, S. T., and Valvano, M. A. (2005). An expression vector containing a rhamnose-inducible promoter provides tightly regulated gene expression in 
Burkholderia cenocepacia. Plasmid 54, 219-228. doi: 10.1016/j.plasmid.2005. 03.004

Carver, T., Berriman, M., Tivey, A., Patel, C., Bohme, U., Barrell, B. G., et al. (2008). Artemis and ACT: viewing, annotating and comparing sequences stored in a relational database. Bioinformatics 24, 2672-2676. doi: 10.1093/bioinformatics/ btn529

Chung, H. S., and Raetz, C. R. (2011). Dioxygenases in Burkholderia ambifaria and Yersinia pestis that hydroxylate the outer Kdo unit of lipopolysaccharide. Proc. Natl. Acad. Sci. U.S.A. 108, 510-515. doi: 10.1073/pnas.101646 2108

Chung, H. S., Yang, E. G., Hwang, D., Lee, J. E., Guan, Z., and Raetz, C. R. (2014). Kdo hydroxylase is an inner core assembly enzyme in the Ko-containing lipopolysaccharide biosynthesis. Biochem. Biophys. Res. Commun. 452, 789-794. doi: 10.1016/j.bbrc.2014.08.153

Cigana, C., Curcuru, L., Leone, M. R., Ierano, T., Lore, N. I., Bianconi, I., et al. (2009). Pseudomonas aeruginosa exploits lipid A and muropeptides modification as a strategy to lower innate immunity during cystic fibrosis lung infection. PLoS ONE 4:e8439. doi: 10.1371/journal.pone.0008439

Ciucanu, I., and Kerek, F. (1984). A simple and rapid method for the permethylation of carbohydrates. Carbohydr. Res. 131, 209-217. doi: 10.1016/ 0008-6215(84)85242-8

Coburn, B., Wang, P. W., Diaz Caballero, J., Clark, S. T., Brahma, V., Donaldson, S., et al. (2015). Lung microbiota across age and disease stage in cystic fibrosis. Sci. Rep. 5:10241. doi: 10.1038/srep10241

Coutinho, C. P., de Carvalho, C. C., Madeira, A., Pinto-de-Oliveira, A., and Sa-Correia, I. (2011a). Burkholderia cenocepacia phenotypic clonal variation during a 3.5 -year colonization in the lungs of a cystic fibrosis patient. Infect. Immun. 79, 2950-2960. doi: 10.1128/IAI.01366-10

Coutinho, C. P., Dos Santos, S. C., Madeira, A., Mira, N. P., Moreira, A. S., and Sá-Correia, I. (2011b). Long-term colonization of the cystic fibrosis lung by Burkholderia cepacia complex bacteria: epidemiology, clonal variation, and genome-wide expression alterations. Front. Cell. Infect. Microbiol. 1:12. doi: $10.3389 /$ fcimb.2011.00012

Cox, A. D., and Wilkinson, S. G. (1989). Structures of the O-specific polymers from the lipopolysaccharides of the reference strains for Pseudomonas cepacia serogroups O3 and O5. Carbohydr. Res. 195, 123-129. doi: 10.1016/00086215(89)85095-5

Craig, F. F., Coote, J. G., Parton, R., Freer, J. H., and Gilmour, N. J. (1989). A plasmid which can be transferred between Escherichia coli and Pasteurella haemolytica by electroporation and conjugation. J. Gen. Microbiol. 135, 2885-2890. doi: 10.1099/00221287-135-11-2885

Cullen, L., and McClean, S. (2015). Bacterial adaptation during chronic respiratory infections. Pathogens 4, 66-89. doi: 10.3390/pathogens4010066

Cunha, M. V., Leitão, J. H., Mahenthiralingam, E., Vandamme, P., Lito, L., Barreto, C., et al. (2003). Molecular analysis of Burkholderia cepacia complex isolates from a Portuguese cystic fibrosis center: a 7-year study. J. Clin. Microbiol. 41, 4113-4120. doi: 10.1128/JCM.41.9.4113-4120. 2003

De Soyza, A., Ellis, C. D., Khan, C. M., Corris, P. A., and Demarco de Hormaeche, R. (2004). Burkholderia cenocepacia lipopolysaccharide, lipid A, and proinflammatory activity. Am. J. Respir. Crit. Care Med. 170, 70-77. doi: $10.1164 / \mathrm{rccm} .200304-592 \mathrm{OC}$

De Soyza, A., Silipo, A., Lanzetta, R., Govan, J. R., and Molinaro, A. (2008). Chemical and biological features of Burkholderia cepacia complex lipopolysaccharides. Innate Immun. 14, 127-144. doi: 10.1177/ 1753425908093984

Dettman, J. R., Rodrigue, N., Aaron, S. D., and Kassen, R. (2013). Evolutionary genomics of epidemic and nonepidemic strains of Pseudomonas aeruginosa. Proc. Natl. Acad. Sci. U.S.A. 110, 21065-21070. doi: 10.1073/pnas.130786 2110

Döring, G., Parameswaran, I. G., and Murphy, T. F. (2011). Differential adaptation of microbial pathogens to airways of patients with cystic fibrosis and chronic obstructive pulmonary disease. FEMS Microbiol. Rev. 35, 124-146. doi: 10.1111/ j.1574-6976.2010.00237.x

Drevinek, P., and Mahenthiralingam, E. (2010). Burkholderia cenocepacia in cystic fibrosis: epidemiology and molecular mechanisms of virulence. Clin. Microbiol. Infect. 16, 821-830. doi: 10.1111/j.1469-0691.2010.03237.x
D’Souza, J. M., Samuel, G. N., and Reeves, P. R. (2005). Evolutionary origins and sequence of the Escherichia coli O4 O-antigen gene cluster. FEMS Microbiol. Lett. 244, 27-32. doi: 10.1016/j.femsle.2005.01.012

Fathy Mohamed, Y., Hamad, M., Ortega, X. P., and Valvano, M. A. (2017). The LpxL acyltransferase is required for normal growth and penta-acylation of lipid A in Burkholderia cenocepacia. Mol. Microbiol. 104, 144-162. doi: 10.1111/mmi. 13618

Figurski, D. H., and Helinski, D. R. (1979). Replication of an origin-containing derivative of plasmid RK2 dependent on a plasmid function provided in trans. Proc. Natl. Acad. Sci. U.S.A. 76, 1648-1652.

Gronow, S., Noah, C., Blumenthal, A., Lindner, B., and Brade, H. (2003). Construction of a deep-rough mutant of Burkholderia cepacia ATCC 25416 and characterization of its chemical and biological properties. J. Biol. Chem. 278, 1647-1655. doi: 10.1074/jbc.M206942200

Hacker, J., and Carniel, E. (2001). Ecological fitness, genomic islands and bacterial pathogenicity. A Darwinian view of the evolution of microbes. EMBO Rep. 2, 376-381. doi: 10.1093/embo-reports/kve097

Harrison, F. (2007). Microbial ecology of the cystic fibrosis lung. Microbiology 153(Pt 4), 917-923. doi: 10.1099/mic.0.2006/004077-0

Holden, M. T., Seth-Smith, H. M., Crossman, L. C., Sebaihia, M., Bentley, S. D., Cerdeno-Tarraga, A. M., et al. (2009). The genome of Burkholderia cenocepacia J2315, an epidemic pathogen of cystic fibrosis patients. J. Bacteriol. 191, 261-277. doi: 10.1128/JB.01230-08

Hu, B., Perepelov, A. V., Liu, B., Shevelev, S. D., Guo, D., Senchenkova, S. N., et al. (2010). Structural and genetic evidence for the close relationship between Escherichia coli O71 and Salmonella enterica O28 O-antigens. FEMS Immunol. Med. Microbiol. 59, 161-169. doi: 10.1111/j.1574-695X.2010.00676.x

Isshiki, Y., Kawahara, K., and Zahringer, U. (1998). Isolation and characterisation of disodium (4-amino-4-deoxy-beta-L-arabinopyranosyl)(1->8)-(D-glycero-alpha-D-talo-oct-2-ulopyranosylonate)-(2->4)-(methyl 3-deoxy-D-manno-oct-2-ulopyranosid)onate from the lipopolysaccharide of Burkholderia cepacia. Carbohydr. Res. 313, 21-27. doi: 10.1016/S0008-6215(98) 00179-7

Kittelberger, R., and Hilbink, F. (1993). Sensitive silver-staining detection of bacterial lipopolysaccharides in polyacrylamide gels. J. Biochem. Biophys. Methods 26, 81-86. doi: 10.1016/0165-022X(93)90024-I

Langmead, B., and Salzberg, S. L. (2012). Fast gapped-read alignment with Bowtie 2. Nat. Methods 9, 357-359. doi: 10.1038/nmeth.1923

Lee, A. H., Flibotte, S., Sinha, S., Paiero, A., Ehrlich, R. L., Balashov, S., et al. (2017). Phenotypic diversity and genotypic flexibility of Burkholderia cenocepacia during long-term chronic infection of cystic fibrosis lungs. Genome Res. 27, 650-662. doi: 10.1101/gr.213363.116

Lerouge, I., and Vanderleyden, J. (2002). O-antigen structural variation: mechanisms and possible roles in animal/plant-microbe interactions. FEMS Microbiol. Rev. 26, 17-47. doi: 10.1111/j.1574-6976.2002.tb00597.x

$\mathrm{Li}, \mathrm{H}$., and Durbin, R. (2010). Fast and accurate long-read alignment with BurrowsWheeler transform. Bioinformatics 26, 589-595. doi: 10.1093/bioinformatics/ btp698

Lieberman, T. D., Michel, J. B., Aingaran, M., Potter-Bynoe, G., Roux, D., Davis, M. R., et al. (2011). Parallel bacterial evolution within multiple patients identifies candidate pathogenicity genes. Nat. Genet. 43, 1275-1280. doi: $10.1038 /$ ng. 997

Lombardi, A., Andreozzi, C., Pavone, V., Triglione, V., Angiolini, L., and Caccia, P. (2013). Evaluation of the oligosaccharide composition of commercial follicle stimulating hormone preparations. Electrophoresis 34, 2394-2406. doi: 10.1002/ elps.201300045

Lyczak, J. B., Cannon, C. L., and Pier, G. B. (2002). Lung infections associated with cystic fibrosis. Clin. Microbiol. Rev. 15, 194-222. doi: 10.1128/cmr.15.2.194-222. 2002

Madeira, A., dos Santos, S. C., Santos, P. M., Coutinho, C. P., Tyrrell, J., McClean, S., et al. (2013). Proteomic profiling of Burkholderia cenocepacia clonal isolates with different virulence potential retrieved from a cystic fibrosis patient during chronic lung infection. PLOS ONE 8:e83065. doi: 10.1371/ journal.pone. 0083065

Madeira, A., Santos, P. M., Coutinho, C. P., Pinto-de-Oliveira, A., and Sá-Correia, I. (2011). Quantitative proteomics (2-D DIGE) reveals molecular strategies employed by Burkholderia cenocepacia to adapt to the airways of cystic fibrosis 
patients under antimicrobial therapy. Proteomics 11, 1313-1328. doi: 10.1002/ pmic. 201000457

Mahenthiralingam, E., Baldwin, A., and Vandamme, P. (2002). Burkholderia cepacia complex infection in patients with cystic fibrosis. J. Med. Microbiol. 51, 533-538. doi: 10.1099/0022-1317-51-7-533

Mahenthiralingam, E., Coenye, T., Chung, J. W., Speert, D. P., Govan, J. R., Taylor, P., et al. (2000). Diagnostically and experimentally useful panel of strains from the Burkholderia cepacia complex. J. Clin. Microbiol. 38, 910-913.

Mahenthiralingam, E., Urban, T. A., and Goldberg, J. B. (2005). The multifarious, multireplicon Burkholderia cepacia complex. Nat. Rev. Microbiol. 3, 144-156. doi: 10.1038/nrmicro1085

Maldonado, R. F., Sá-Correia, I., and Valvano, M. A. (2016). Lipopolysaccharide modification in Gram-negative bacteria during chronic infection. FEMS Microbiol. Rev. 40, 480-493. doi: 10.1093/femsre/fuw007

Marolda, C. L., Lahiry, P., Vines, E., Saldias, S., and Valvano, M. A. (2006). Micromethods for the characterization of lipid A-core and O-antigen lipopolysaccharide. Methods Mol. Biol. 347, 237-252.

Marolda, C. L., Welsh, J., Dafoe, L., and Valvano, M. A. (1990). Genetic analysis of the O7-polysaccharide biosynthesis region from the Escherichia coli O7:K1 strain VW187. J. Bacteriol. 172, 3590-3599. doi: 10.1128/jb.172.7.3590-3599. 1990

Mira, N. P., Madeira, A., Moreira, A. S., Coutinho, C. P., and Sá-Correia, I. (2011). Genomic expression analysis reveals strategies of Burkholderia cenocepacia to adapt to cystic fibrosis patients' airways and antimicrobial therapy. PLoS ONE 6:e28831. doi: 10.1371/journal.pone.0028831

Moreira, A. S., Lourenco, A. B., and Sá-Correia, I. (2016a). 1H-NMR-based endometabolome profiles of Burkholderia cenocepacia clonal variants retrieved from a cystic fibrosis patient during chronic infection. Front. Microbiol. 7:2024. doi: $10.3389 /$ fmicb.2016.02024

Moreira, A. S., Mil-Homens, D., Sousa, S. A., Coutinho, C. P., Pinto-de-Oliveira, A., Ramos, C. G., et al. (2016b). Variation of Burkholderia cenocepacia virulence potential during cystic fibrosis chronic lung infection. Virulence doi: 10.1080/ 21505594.2016.1237334 [Epub ahead of print].

Nishiyama, E., Ohtsubo, Y., Nagata, Y., and Tsuda, M. (2010). Identification of Burkholderia multivorans ATCC 17616 genes induced in soil environment by in vivo expression technology. Environ. Microbiol. 12, 2539-2558. doi: 10.1111/ j.1462-2920.2010.02227.x

Ortega, X., Hunt, T. A., Loutet, S., Vinion-Dubiel, A. D., Datta, A., Choudhury, B., et al. (2005). Reconstitution of O-specific lipopolysaccharide expression in Burkholderia cenocepacia strain J2315, which is associated with transmissible infections in patients with cystic fibrosis. J. Bacteriol. 187, 1324-1333. doi: 10.1128/JB.187.4.1324-1333.2005

Ortega, X., Silipo, A., Saldias, M. S., Bates, C. C., Molinaro, A., and Valvano, M. A. (2009). Biosynthesis and structure of the Burkholderia cenocepacia K56-2 lipopolysaccharide core oligosaccharide: truncation of the core oligosaccharide leads to increased binding and sensitivity to polymyxin B. J. Biol. Chem. 284, 21738-21751. doi: 10.1074/jbc.M109.008532

Piantini, U., Sorensen, O. W., and Ernst, R. R. (1982). Multiple Quantum Filters for Elucidating NMR Coupling Networks. J. Am. Chem. Soc. 104, 6800-6801. doi: 10.1021/ja00388a062

Raetz, C. R., and Whitfield, C. (2002). Lipopolysaccharide endotoxins. Annu. Rev. Biochem. 71, 635-700. doi: 10.1146/annurev.biochem.71.110601.135414

Rance, M., Sørensen, O. W., Bodenhausen, G., Wagner, G., Ernst, R. R., and Wüthrich, K. (1983). Improved spectral resolution in cosy 1H NMR spectra of proteins via double quantum filtering. Biochem. Biophys. Res. Commun. 117, 479-485. doi: 10.1016/0006-291X(83)91225-1

Reeves, P. R., Cunneen, M. M., Liu, B., and Wang, L. (2013). Genetics and evolution of the Salmonella galactose-initiated set of o antigens. PLoS ONE 8:e69306. doi: 10.1371/journal.pone.0069306

Rietschel, E. T. (1976). Absolute-Configuration of 3-Hydroxy Fatty-Acids Present in Lipopolysaccharides from Various Bacterial Groups. Eur. J. Biochem. 64, 423-428. doi: 10.1111/j.1432-1033.1976.tb10318.x

Robinson, J. T., Thorvaldsdottir, H., Winckler, W., Guttman, M., Lander, E. S., Getz, G., et al. (2011). Integrative genomics viewer. Nat. Biotechnol. 29, 24-26. doi: $10.1038 /$ nbt. 1754

Saldias, M. S., Ortega, X., and Valvano, M. A. (2009). Burkholderia cenocepacia $\mathrm{O}$ antigen lipopolysaccharide prevents phagocytosis by macrophages and adhesion to epithelial cells. J. Med. Microbiol. 58(Pt 12), 1542-1548. doi: 10.1099/jmm.0.013235-0

Seemann, T. (2014). Prokka: rapid prokaryotic genome annotation. Bioinformatics 30, 2068-2069. doi: 10.1093/bioinformatics/btu153

Silipo, A., Molinaro, A., Cescutti, P., Bedini, E., Rizzo, R., Parrilli, M., et al. (2005). Complete structural characterization of the lipid A fraction of a clinical strain of B. cepacia genomovar I lipopolysaccharide. Glycobiology 15, 561-570. doi: 10.1093/glycob/cwi029

Silipo, A., Molinaro, A., Ierano, T., De Soyza, A., Sturiale, L., Garozzo, D., et al. (2007). The complete structure and pro-inflammatory activity of the lipooligosaccharide of the highly epidemic and virulent gram-negative bacterium Burkholderia cenocepacia ET-12 (strain J2315). Chemistry 13, 3501-3511. doi: 10.1002/chem.200601406

Silva, I. N., Santos, P. M., Santos, M. R., Zlosnik, J. E., Speert, D. P., Buskirk, S. W., et al. (2016). Long-Term Evolution of Burkholderia multivorans during a Chronic Cystic Fibrosis Infection Reveals Shifting Forces of Selection. mSystems 1, e29-16. doi: 10.1128/mSystems.00029-16

Spencer, D. H., Kas, A., Smith, E. E., Raymond, C. K., Sims, E. H., Hastings, M., et al. (2003). Whole-genome sequence variation among multiple isolates of Pseudomonas aeruginosa. J. Bacteriol. 185, 1316-1325. doi: 10.1128/JB.185.4. 1316-1325.2003

States, D. J., Haberkorn, R. A., and Ruben, D. J. (1982). A two-dimensional nuclear overhauser experiment with pure absorption phase in four quadrants. J. Magn. Reson. 48, 286-292. doi: 10.1016/0022-2364(82)90279-7

Stern, A. S., Li, K. B., and Hoch, J. C. (2002). Modern spectrum analysis in multidimensional NMR spectroscopy: comparison of linear-prediction extrapolation and maximum-entropy reconstruction. J. Am. Chem. Soc. 124, 1982-1993. doi: 10.1021/ja011669o

Sturiale, L., Garozzo, D., Silipo, A., Lanzetta, R., Parrilli, M., and Molinaro, A. (2005). New conditions for matrix-assisted laser desorption/ionization mass spectrometry of native bacterial R-type lipopolysaccharides. Rapid Commun. Mass Spect. 19, 1829-1834. doi: 10.1002/rcm.1994

Thrane, S. W., Taylor, V. L., Freschi, L., Kukavica-Ibrulj, I., Boyle, B., Laroche, J., et al. (2015). The Widespread Multidrug-Resistant Serotype O12 Pseudomonas aeruginosa Clone Emerged through Concomitant Horizontal Transfer of Serotype Antigen and Antibiotic Resistance Gene Clusters. MBio 6, e1396-15. doi: 10.1128/mBio.01396-15

Traverse, C. C., Mayo-Smith, L. M., Poltak, S. R., and Cooper, V. S. (2013). Tangled bank of experimentally evolved Burkholderia biofilms reflects selection during chronic infections. Proc. Natl. Acad. Sci. U.S.A. 110, E250-E259. doi: 10.1073/ pnas. 1207025110

Valvano, M. A. (2003). Export of O-specific lipopolysaccharide. Front. Biosci. 8:s452-s471. doi: 10.2741/1079

Valvano, M. A. (2015). "Genetics and biosynthesis of lipoplysaccharide," in Molecular Medical Microbiology, 2nd Edn, eds Y.-W. Tang, M. Sussman, L. Dongyou, I. Poxton, and J. Schwartzman (Amsterdam: Elsevier), 55-89.

Valvano, M. A., Furlong, S. E., and Patel, K. B. (2011). "Genetics, biosynthesis and assembly of O-Antigen," in Bacterial Lipopolysaccharides, eds Y. A. Knirel and M. A. Valvano (Wien: Springer-Verlag), 275-310.

Vandamme, P., Holmes, B., Vancanneyt, M., Coenye, T., Hoste, B., Coopman, R., et al. (1997). Occurrence of multiple genomovars of Burkholderia cepacia in cystic fibrosis patients and proposal of Burkholderia multivorans sp. nov. Int. J. Syst. Bacteriol. 47, 1188-1200. doi: 10.1099/00207713-47-41188

Westphal, O., and Jann, K. (1965). "Bacterial lipopolysaccharides: extraction with phenol-water and further applications of the procedure," in Methods in Carbohydrate Chemistry, eds R. L. Whistler and M. L. Wolfrom (New York, NY: Academic Press), 83-91.

Whitfield, C., and Trent, M. S. (2014). Biosynthesis and export of bacterial lipopolysaccharides. Annu. Rev. Biochem. 83, 99-128. doi: 10.1146/annurevbiochem-060713-035600

Yang, L., Jelsbak, L., Marvig, R. L., Damkiaer, S., Workman, C. T., Rau, M. H., et al. (2011). Evolutionary dynamics of bacteria in a human host environment. Proc. Natl. Acad. Sci. U.S.A. 108, 7481-7486. doi: 10.1073/pnas.101824 9108

Zerbino, D. R., and Birney, E. (2008). Velvet: algorithms for de novo short read assembly using de Bruijn graphs. Genome Res. 18, 821-829. doi: 10.1101/gr. 074492.107 
Zhang, R., Ou, H. Y., Gao, F., and Luo, H. (2014). Identification of horizontally-transferred genomic islands and genome segmentation points by using the gc profile method. Curr. Genomics 15, 113-121. doi: 10.2174/ 1389202915999140328163125

Conflict of Interest Statement: The authors declare that the research was conducted in the absence of any commercial or financial relationships that could be construed as a potential conflict of interest.
Copyright (C) 2017 Hassan, Maldonado, dos Santos, Di Lorenzo, Silipo, Coutinho, Cooper, Molinaro, Valvano and Sá-Correia. This is an open-access article distributed under the terms of the Creative Commons Attribution License (CC BY). The use, distribution or reproduction in other forums is permitted, provided the original author(s) or licensor are credited and that the original publication in this journal is cited, in accordance with accepted academic practice. No use, distribution or reproduction is permitted which does not comply with these terms. 